\title{
Wavevector-Dependent Susceptibility in $Z$-Invariant Pentagrid Ising Model
}

\author{
Helen Au-Yang and Jacques H.H. Perk ${ }^{1,2}$
}

Received September 17, 2004

\begin{abstract}
We study the $\mathbf{q}$-dependent susceptibility $\chi(\mathbf{q})$ of a $Z$-invariant ferromagnetic Ising model on a Penrose tiling, as first introduced by Korepin using de Bruijn's pentagrid for the rapidity lines. The pair-correlation function for this model can be calculated exactly using the quadratic difference equations from our previous papers. Its Fourier transform $\chi(\mathbf{q})$ is studied using a novel way to calculate the joint probability for the pentagrid neighborhoods of the two spins, reducing this calculation to linear programming. Since the lattice is quasiperiodic, we find that $\chi(\mathbf{q})$ is aperiodic and has everywhere dense peaks, which are not all visible at very low or high temperatures. More and more peaks become visible as the correlation length increases - that is, as the temperature approaches the critical temperature.
\end{abstract}

KEY WORDS: Ising model; quasiperiodicity; Fibonacci sequence; pentagrid; Penrose tiles; $Z$-invariance; correlation functions; q-dependent susceptibility.

\section{Introduction}

In an experiment ${ }^{(1)}$ done in 1984, Shechtman and his coworkers found fivefold symmetry in the diffraction patterns of some rapidly cooled alloys. As such a symmetry is incompatible with lattice periodicity, it was concluded that the crystalline structures of these alloys, if any, must necessarily be quasiperiodic. This theoretical explanation came forward almost immediately, as Penrose, de Bruijn, and Mackay ${ }^{(2,3,4] 5,6,7)}$ had already studied tilings that have fivefold symmetry, well before this experimental discovery. Quasiperiodic tilings are

\footnotetext{
${ }^{1}$ Department of Physics, Oklahoma State University, Stillwater, OK 74078-3072, USA.

${ }^{2}$ Supported in part by NSF Grant No. PHY 01-00041.
} 
types of almost periodic structures that permit sharp peaks in the diffraction patterns, but have normally forbidden symmetries. $.7,8,9,10,11,12)$

Already in 1986, Korepin ${ }^{(13)}$ introduced a $Z$-invariant eight-vertex model on Penrose tiles. The $Z$-invariant inhomogeneous models are completely integrable ${ }^{(14)}$ even on irregular lattices and their critical exponents are known to be the same as those of homogeneous systems on regular lattices. Thus, the critical behaviors of these quasiperiodic $Z$-invariant models $(14], 15,16] 17,19,20,21,18)$ have to be the same 3 independent of the lattice structure.4

In such models, the order parameter is the same $\mathrm{e}^{(14] 13)}$ for all sites and it vanishes towards the critical point. Therefore, the Fourier transform of the one-point function of a $Z$-invariant Ising model is the product of this order parameter and the lattice sum $\sum \mathrm{e}^{\mathrm{iq} \cdot \mathbf{r}}$. Experiments that probe the resulting "magnetic" Bragg peaks are restricted to the low-temperature phase and the corresponding theory is essentially the zero-temperature theory, well-studied in the literature. ${ }^{(7 \sqrt[37]{38})}$ The aforementioned Bragg peaks will broaden, if we allow the underlying quasicrystalline lattice to become distorted by lattice vibrations. (39,40) However, we shall not consider this possibility in this paper, as we assume the underlying lattice to be a perfect and rigid Penrose tiling, restricting our attention solely to the ordering of the spins at the lattice sites under thermal fluctuations.

Contrary to the above theory for the Bragg scattering, the situation is far more complicated for scattering experiments that probe the pair-correlation function $\left\langle\sigma_{\mathbf{r}} \sigma_{\mathbf{r}^{\prime}}\right\rangle$ via the wavevector-dependent susceptibility $\chi(\mathbf{q})$. This last quantity is defined as

$$
k_{\mathrm{B}} T \chi(\mathbf{q}) \equiv \bar{\chi}(\mathbf{q})=\lim _{\mathcal{L} \rightarrow \infty} \frac{1}{\mathcal{L}} \sum_{\mathbf{r}} \sum_{\mathbf{r}^{\prime}} \mathrm{e}^{\mathrm{i} \mathbf{q} \cdot\left(\mathbf{r}^{\prime}-\mathbf{r}\right)}\left[\left\langle\sigma_{\mathbf{r}} \sigma_{\mathbf{r}^{\prime}}\right\rangle-\left\langle\sigma_{\mathbf{r}}\right\rangle\left\langle\sigma_{\mathbf{r}^{\prime}}\right\rangle\right]
$$

where $\mathcal{L}$ is the number of lattice sites, $\mathbf{r}$ and $\mathbf{r}^{\prime}$ run through all these sites, and $\mathbf{q}=\left(q_{x}, q_{y}\right)$. It is the Fourier transform of the connected pair correlation function, defined by what is inside the square brackets. Furthermore, $\bar{\chi}(\mathbf{q})$ is

\footnotetext{
${ }^{3}$ Universality of the critical exponents of ferromagnetic Ising models on quasiperiodic lattices has been confirmed for non- $Z$-invariant cases also using real-space renormaliza-

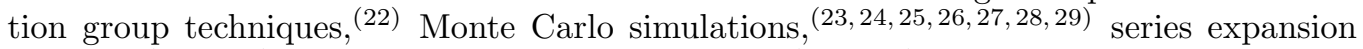
methods, $\stackrel{(30|31| 32)}{32}$ and the study of Yang-Lee zeros. $.33|34| 35)$

${ }^{4}$ Unlike the q-dependent susceptibility, thermodynamic quantities like the free energy, the specific heat, and the bulk susceptibility do not probe the lattice structure, although subtle lattice effects do show up in corrections to scaling. ${ }^{(36)}$
} 
the reduced $\mathbf{q}$-dependent susceptibility, taking out a trivial factor involving the absolute temperature $T$.

In the well-known lattice-gas language it becomes proportional to the structure function, the Fourier transform of the density-density correlation function, which is also measurable in diffraction experiments, revealing the symmetry of the lattice. Thus, the $\chi(\mathbf{q})$ in $Z$-invariant models can indeed be used to show the difference between quasiperiodic and regular lattices and we expect it to provide a diffuse scattering pattern both above and below the critical temperature $T_{\mathrm{c}}$, with more structure closer to $T_{\mathrm{c}}$.

There is good reason to pick a $Z$-invariant Ising model for our present study of $\chi(\mathbf{q})$. It is taken from the foremost class of models with short-range interactions allowing exact computations. Moreover, comprehensive extensive studies on spin-spin correlation functions in nontrivial models with shortrange interactions have been done only for Ising models. $41,42,43,44,45,46,47,14], 48)$ Cited here is just a fraction of the literature. The accumulative knowledge of these studies has made the calculations in $Z$-invariant quasi-periodic Ising models possible. Correlations in other nontrivial cases are still mostly inaccessible to exact methods of evaluation.

For instance, in 1988, Tracy $(\underline{40 / 50})$ introduced the layered Fibonacci Ising model, which is not $Z$-invariant. The row correlation functions in the layered Ising model are known to be block Toeplitz determinants, ${ }^{(51)}$ of which we still have no idea how to evaluate them exactly in general, except in a few simpler cases. 51 (52) Tracy has shown that the critical exponent of the specific heat remains unchanged in such quasiperiodic layered models.

In our previous papers, ${ }^{(53[54)}$ we have studied Fibonacci Ising models whose spins are on regular lattices, but whose nearest-neighbor interactions are quasi-periodic. They are special cases of inhomogeneous Ising models whose Hamiltonians are given by

$$
-\beta \mathcal{H}=\sum_{m, n}\left(\bar{K}_{m, n} \sigma_{m, n} \sigma_{m, n+1}+K_{m, n} \sigma_{m, n} \sigma_{m+1, n}\right),
$$

with $\beta \equiv 1 / k_{\mathrm{B}} T$. When the system is periodic, the pair correlations are translationally invariant. Thus one of the sums in (1.1) can be carried out. For $T \neq T_{c}$, the connected correlations decay exponentially as functions of distance 5 Therefore, only a finite number of short-distance correlation functions are needed for the calculation of the $\chi(\mathbf{q})$ in Eq. (1.1).

\footnotetext{
${ }^{5}$ For $T=T_{\mathrm{c}}$, the correlations decay algebraically, so that we need the Epstein-Ewald
} summation formula $\frac{(55)}{5}$ to take into account the long-distance behavior. 


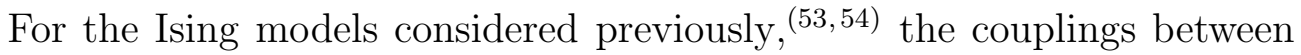
specific nearest-neighbor spin pairs form Fibonacci sequences $\left\{S_{n}\right\}$ defined recursively $\stackrel{(49)}{ }$ by

$$
S_{n+1}=S_{n} S_{n-1}, \quad S_{0}=\mathrm{B}, \quad S_{1}=\mathrm{A},
$$

so that $S_{2}=\mathrm{AB}, S_{3}=\mathrm{ABA}, S_{4}=\mathrm{ABAAB}$ and so on. Since this sequence is quasiperiodic, as arbitrary long subsequences are repeated infinitely often, the model is also aperiodic. Consequently, the correlations are no longer translationally invariant. However, the averages of the correlations for two spins at fixed distance can be evaluated by using a theorem of Tracy. (49)

In our previous works, ${ }^{(53,54)}$ we have found that the various q-dependent susceptibilities $\chi(\mathbf{q})$ of our Fibonacci Ising models are always periodic. They can have multiple incommensurate everywhere-dense peaks in each unit cell only if the aperiodic oscillations in the average correlation functions are not negligibly small. This is true in the mixed case when the interactions are aperiodic sequences of ferromagnetic and antiferromagnetic couplings. The number of visible peaks in $\chi(\mathbf{q})$ increases as the correlation length increases. In contrast, in the periodic Fibonacci Ising lattices with mixed bonds, the visible peaks in the $\mathbf{q}$-dependent susceptibility are at commensurate positions and their number has a finite maximum.

The ferromagnetic aperiodic Fibonacci Ising lattice, on the other hand, behaves almost like the regular Ising model - one peak per unit cell, located at the commensurate position - because the aperiodic oscillations in its average correlation functions are negligibly small at all temperatures. ${ }^{(53)}$

The q-dependent susceptibility $\chi(\mathbf{q})$ is found to have almost the same behaviors for both $T<T_{\mathrm{c}}$ and $T>T_{\mathrm{c}}{ }^{\left({ }^{(53)}\right)}$ This is in agreement with the result of Peter Stephens, who showed that the randomized (disordered) icosahedral system ${ }^{(56)}$ gives almost the same diffraction pattern as a quasicrystal — which is in the solid (ordered) phase.

Even though both ferromagnetic and antiferromagnetic edge interactions are present in the mixed case, the mixed systems in Refs. 53 and 54 are not frustrated. In fact, their partition functions are equal to the partition functions of the ferromagnetic models, from which they differ by gauge transformations of signs. Also, the $\chi(\mathbf{q})$ of a fully-frustrated model may not show incommensurate peaks. ${ }^{(55)}$

In this paper, we turn our attention to systems with a quasiperiodic lattice structure. (19,20]21) More specifically we study a $Z$-invariant Ising model whose 
spins are on vertices of a Penrose fat-and-skinny rhombus tiling with the pentagrid as its rapidity lines. $\frac{(5,13,15,16,17)}{17}$

\subsection{Outline}

This paper is organized as follows. In Section 2, we introduce a $Z$-invariant Ising model on Penrose tiles constructed from a pentagrid, (5) with spins on either the odd or even sublattice. We show how the pair-correlation functions can be evaluated in Section 3. Section 4 is rather lengthy. In it, a new method of counting all the spin sites and evaluating the joint probabilities of the occurrence of two neighborhoods of two spins is given. In subsection 5.1, we describe in detail the calculation for the q-dependent susceptibility for the odd lattice. The results are given in subsection 5.2. In subsection 5.3 we present a mapping between the odd and the even sublattices. Finally, in Section 6 we present our conclusions.

\section{Pentagrid and Penrose Tiles}

In two ingenious papers by the famous Dutch mathematician N.G. de Bruijn, he relates the non-periodic Penrose tilings in a plane to a pentagrid, (5) which is a superposition of five grids. Each grid consists of parallel lines with equal spacings between the lines; the grids may be obtained from one another by rotations of angles which are multiples of $2 \pi / 5$. This is shown in Fig. 1. Here some grid lines of the pentagrid are shown; the arrows on the lines shown in Fig. 1 should be ignored for the moment, as they will define the direction of the "rapidities" which we define later.

To describe the pentagrid in mathematical formulas, ${ }^{(5)}$ let

$$
\zeta=\mathrm{e}^{2 \mathrm{i} \pi / 5}, \quad \zeta+\zeta^{-1}=2 \cos (2 \pi / 5)=p^{-1}=\frac{1}{2}(\sqrt{5}-1),
$$

in which $p$ is the golden ratio. Then, choose $\gamma_{0}, \gamma_{1}, \gamma_{2}, \gamma_{3}, \gamma_{4}$ to be five real numbers, satisfying

$$
\gamma_{0}+\gamma_{1}+\gamma_{2}+\gamma_{3}+\gamma_{4}=0
$$

Now the $j$ th grid in the pentagrid consists of lines given by

$$
G_{j}=\left\{z \in \mathbb{C} \mid \operatorname{Re}\left(z \zeta^{-j}\right)+\gamma_{j}=k_{j}, k_{j} \in \mathbb{Z}\right\}, \quad j=0, \cdots, 4 .
$$

The pentagrid is called regular, if there is no point in the complex plane $\mathbb{C}$ belonging to more than two of the five grids. This also means, every vertex 


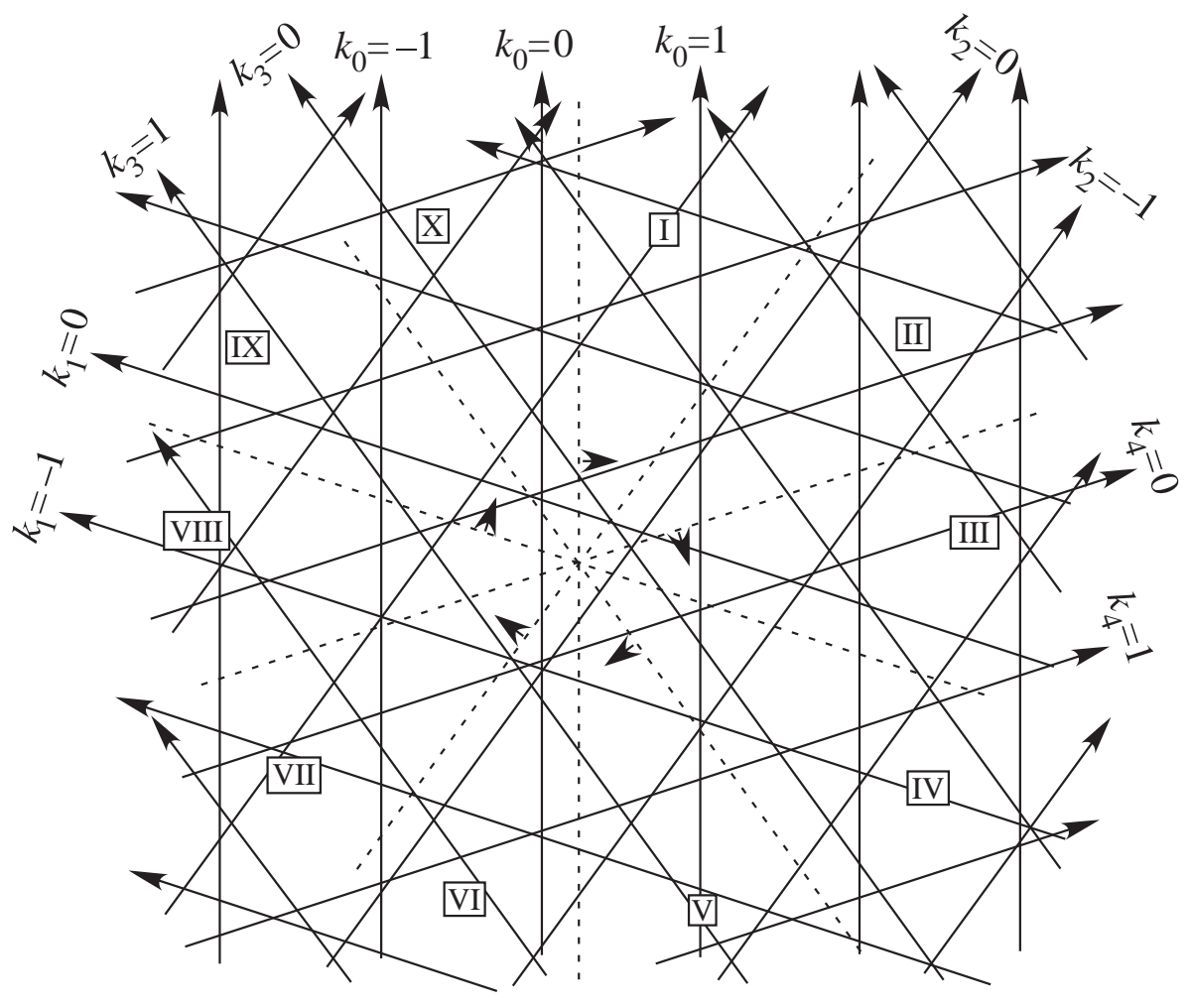

Fig. 1. The pentagrid is a superposition of five grids, each of which consists of parallel equidistanced lines. These grid lines are the five different kinds of rapidity lines in a $Z$ invariant Ising model. Arrows are put on the grid lines, and all of them pointing to the upper half plane. Through a center in one of the meshes (site for a spin $\sigma$ ), dashed lines are drawn to split the pentagrid into ten regions I to X. Correlations between $\sigma$ and $\sigma^{\prime}$ are different when $\sigma^{\prime}$ sits in different regions. Five little arrows perpendicular to the five dashed lines indicate the directions in which the corresponding integers $k_{j}, j=0, \cdots, 4$, increase. 
of the regular pentagrid is an intersection of no more than two lines. Each vertex is surrounded by four meshes (which are often called faces in physics).

Now to every point $z$ in the complex plane $\mathbb{C}$, de Bruijn associates an integer vector $\vec{K}(z)=\left(K_{0}(z), \cdots, K_{4}(z)\right)$ whose five elements are integers given by

$$
K_{j}(z)=\left\lceil\operatorname{Re}\left(z \zeta^{-j}\right)+\gamma_{j}\right\rceil
$$

in which $\lceil x\rceil$ denotes the "roof of $x$ ", which is the smallest integer $\geqslant x$. It is easily seen from (2.4) and (2.3) that whenever $z$ moves across a line of the $j$ th grid, $K_{j}(z)$ changes by 1 . All points in the same mesh (face) have the same integer vector and the integer vectors of different meshes are different. From Fig. 1, we may already see that some fraction of the meshes (or faces) becomes infinitesimally small in size as the number of lines in each grid becomes infinite.

Since every vertex of the regular pentagrid is surrounded by four meshes (faces), by assigning to each of their four corresponding integer vectors $\vec{K}(z)$ $=\left(K_{0}(z), \cdots, K_{4}(z)\right)$ a complex number

$$
f(z)=\sum_{j=0}^{4} K_{j}(z) \zeta^{j},
$$

these four meshes are now mapped to the vertices of a rhombus. More specifically, to the intersection of two grid lines $k_{r}$ and $k_{s},(r \neq s)$, one assigns a rhombus in $\mathbb{C}$ whose vertices are the four complex numbers $f(z)$, $f(z)+\zeta^{r}, f(z)+\zeta^{s}$ and $f(z)+\zeta^{r}+\zeta^{s}$ assigned to the four surrounding meshes. Clearly, there are two different kinds of rhombuses: the thick one having angles $72^{\circ}$ and $108^{\circ}$ for $r=s \pm 1$, and the thin one having angles $36^{\circ}$ and $144^{\circ}$ for $r=s \pm 2$. In both rhombuses, all sides have length 1 . They are shown in Fig. 2.

In these two papers, ${ }^{(5)}$ de Bruijn also showed that, even though there are many different choices of $\gamma_{j}$ in (2.2), many of the resulting pentagrids are shift-equivalent, that is, they can be obtained from each other by a parallel shift 6

We now assign to each grid line in the grid $j, j=0, \cdots, 4$, of the pentagrid a rapidity $u_{j}$ pointing into the upper half plane, as is shown in Figs. 1 and

$\overline{{ }^{6} \mathrm{He} \text { also }}$ proved that if $\xi \equiv \sum_{j} \gamma_{j} \zeta^{2 j}$ times a power of $\zeta$ is not purely imaginary (modulo the principal ideal of $1-\zeta$ given by all complex numbers of the form $\sum_{j} n_{j} \zeta^{j}$ with integers $n_{0}, \cdots, n_{4}$ satisfying $\left.\sum_{j} n_{j}=0\right)$, then the corresponding pentagrid is regular. $\underline{(5)}$ 


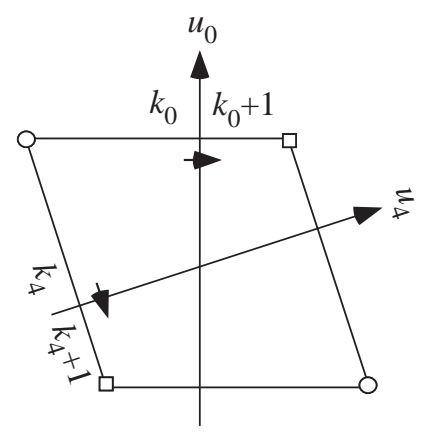

(a) Thick rhombus

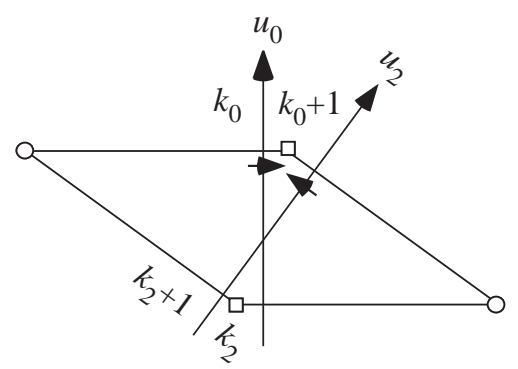

(b) Thin rhombus

Fig. 2. Spins interacts along the diagonals. All sides have length 1. The long diagonal of the thick rhombus has length $p$ and the short diagonal of the thin rhombus has length $p^{-1}$, with $p$ the golden ratio, given in (2.1). The little arrows perpendicular to the grid (rapidity) lines indicate the directions in which the integers $k_{j}, j=0, \cdots, 4$, increase.

3. In Fig. 3, it is also shown how the five grid lines $k_{j}=0$, for $j=0, \cdots, 4$, are shifted to make the grid regular, again following de Bruijn. ${ }^{(5)}$

The usual $Z$-invariant Ising model ${ }^{(14 \sqrt[48)]{4}}$ is formed by putting spins inside the meshes, but here, however, the Ising spins are on the vertices of the rhombuses. There is an one-to-one mapping given by (2.5) relating a mesh in the pentagrid to a vertex of the Penrose tiling. After the mapping, the grid lines in the pentagrid - which are also the rapidity lines - become "Conway worms" (no longer straight) in the Penrose tiling. (57) Since the rapidity lines are used to define commuting transfer matrices, they do not have to be straight lines.

The model so defined is a special case of the inhomogeneous $Z$-invariant eight-vertex model proposed by Korepin. (13]15],16]17) More specifically, the four-spin couplings are identically zero, and the eight-vertex model decomposes into two independent Ising models. The interactions of the Ising spins are along the diagonals of the rhombuses. The odd and even sublattices are therefore decoupled..$\left(\frac{58 \mid(59)}{}\right.$

As in the earlier works, ${ }^{(14 \mid 48)}$ the coupling $K\left(u_{i}, v_{j}\right)$ between two spins is represented by a line connecting these two spins, with the arrows of the two

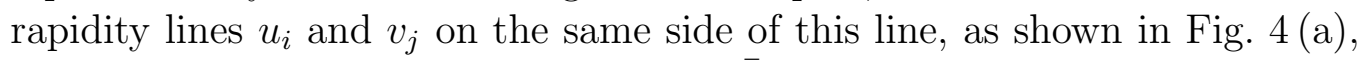
while the line representing the coupling $\bar{K}\left(u_{i}, v_{j}\right)$ has the arrows of the two

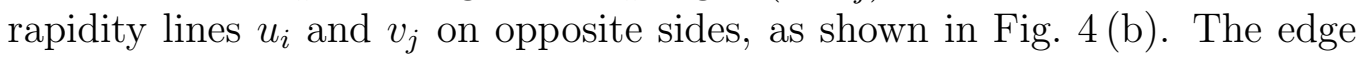




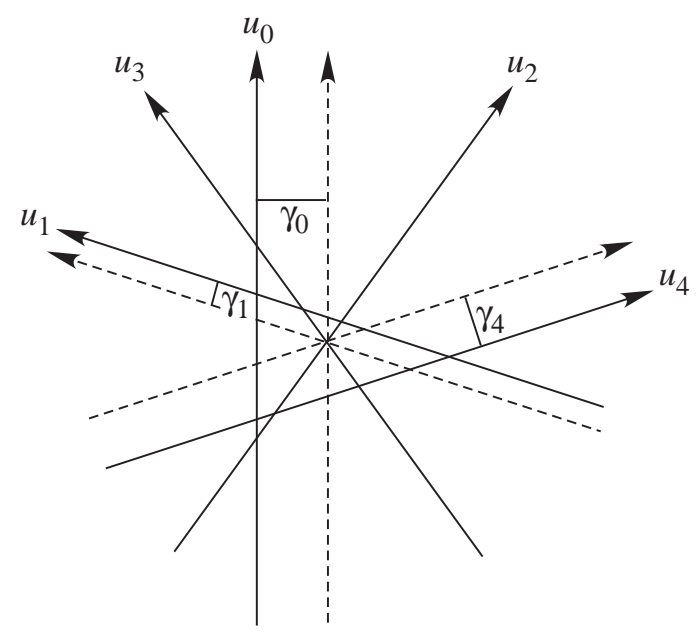

Fig. 3. The five different kinds of rapidity lines $u_{0}, \cdots, u_{4}$ pointing into the upper half plane. The grid lines $k_{j}=0$, for $j=0, \cdots, 4$, are shifted from the dashed lines to make the pentagrid regular.

interactions are parametrized by

$$
\begin{aligned}
& \sinh \left(2 K\left(u_{i}, v_{j}\right)\right)=k \operatorname{sc}\left(u_{i}-v_{j}, k^{\prime}\right)=\operatorname{cs}\left(\lambda+v_{j}-u_{i}, k^{\prime}\right) \\
& \sinh \left(2 \bar{K}\left(u_{i}, v_{j}\right)\right)=\operatorname{cs}\left(u_{i}-v_{j}, k^{\prime}\right)=k \operatorname{sc}\left(\lambda+v_{j}-u_{i}, k^{\prime}\right) .
\end{aligned}
$$

Here $\lambda=\mathrm{K}\left(k^{\prime}\right)$ is the elliptic integral of the first kind, and $k$ and $k^{\prime}=\sqrt{1-k^{2}}$ are the elliptic moduli; these are convenient temperature variables, assumed to be the same for all sites.

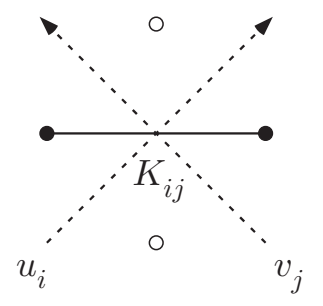

(a)

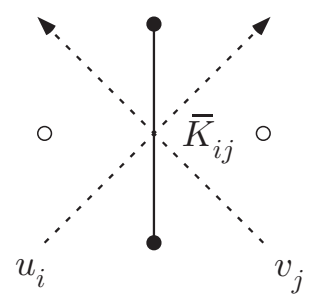

(b)

Fig. 4. (a) The horizontal coupling $K\left(u_{i}, v_{j}\right)$; and (b) the vertical coupling $\bar{K}\left(u_{i}, v_{j}\right)$. 
From Fig. 2, we can see that the lengths of the four diagonals of the two rhombuses are different. The interactions between the spins are chosen to depend on the interparticle spacings only, but not on the orientations. Consequently, we must have

$$
u_{0}-u_{1}=u_{2}-u_{3}=u_{4}-u_{0}=\lambda+u_{1}-u_{2}=\lambda+u_{3}-u_{4} .
$$

From this, we find

$$
u_{4}-u_{1}=\frac{4 \lambda}{5}, \quad u_{2}-u_{1}=\frac{3 \lambda}{5}, \quad u_{0}-u_{1}=\frac{2 \lambda}{5}, \quad u_{3}-u_{1}=\frac{\lambda}{5} .
$$

If we let

$$
s_{j}=k \operatorname{sc}\left(j \lambda / 5, k^{\prime}\right),
$$

then for the thick rhombus in Fig. 2(a), we assign $s_{2}$ to the longer diagonal and $s_{3}$ to the shorter diagonal, while for the thin rhombus in Fig. 2(b), $s_{4}$ to the shorter diagonal and $s_{1}$ to the longer one. Thus, to the four types of diagonals are assigned four kinds of couplings according to their lengths, with a stronger coupling for a shorter interparticle distance.

The two Ising sublattices on the Penrose tiling are indicated in Fig. 5. The edges in the even sublattice are omitted. There are eight types of vertices S, K, Q, D, J, S3, S4, S5 in the Penrose tiling, which are shown in Fig. 7 of Ref. 5. The coordination numbers of spins in the Penrose Ising model are 3 for types Q and D; 4 for K; 5 for S, J and S5; 6 for S4; 7 for S5.

\section{Correlations}

Two spins $\sigma_{\mathbf{r}}$ and $\sigma_{\mathbf{r}^{\prime}}$ at two different vertices of the Ising lattice just defined have different integer vectors $\vec{K}=\left(K_{0}, \cdots, K_{4}\right)$ and $\vec{K}^{\prime}=\left(K_{0}^{\prime}, \cdots, K_{4}^{\prime}\right)$. Since there is a one-to-one mapping between the vertices of the Penrose tiles and the meshes of the pentagrid, the integer vectors can be used to denote the positions of the spins: $\mathbf{r} \leftrightarrow \vec{K}$.

Since each grid consists of parallel lines with equal spacings, the absolute value of the difference $\ell_{j}=K_{j}^{\prime}-K_{j}$ is actually the number of the $j$ th kind of rapidity lines sandwiched between these two spins. The correlation functions were shown $\stackrel{(14,48)}{40}$ be

$$
\begin{aligned}
& \left\langle\sigma_{\vec{K}} \sigma_{\vec{K}^{\prime}}\right\rangle=\left\langle\sigma \sigma^{\prime}\right\rangle_{\left[\ell_{0}, \cdots, \ell_{4}\right]} \\
& =g(\overbrace{u_{0}^{\prime}, \ldots, u_{0}^{\prime}}^{\left|\ell_{0}\right|} \overbrace{u_{1}^{\prime}, \ldots, u_{1}^{\prime}}^{\left|\ell_{1}\right|} \overbrace{u_{2}^{\prime}, \ldots, u_{2}^{\prime}}^{\left|\ell_{2}\right|}, \overbrace{u_{3}^{\prime}, \ldots, u_{3}^{\prime}}^{\left|\ell_{3}\right|} \overbrace{u_{4}^{\prime}, \ldots, u_{4}^{\prime}}^{\left|\ell_{4}\right|}),
\end{aligned}
$$




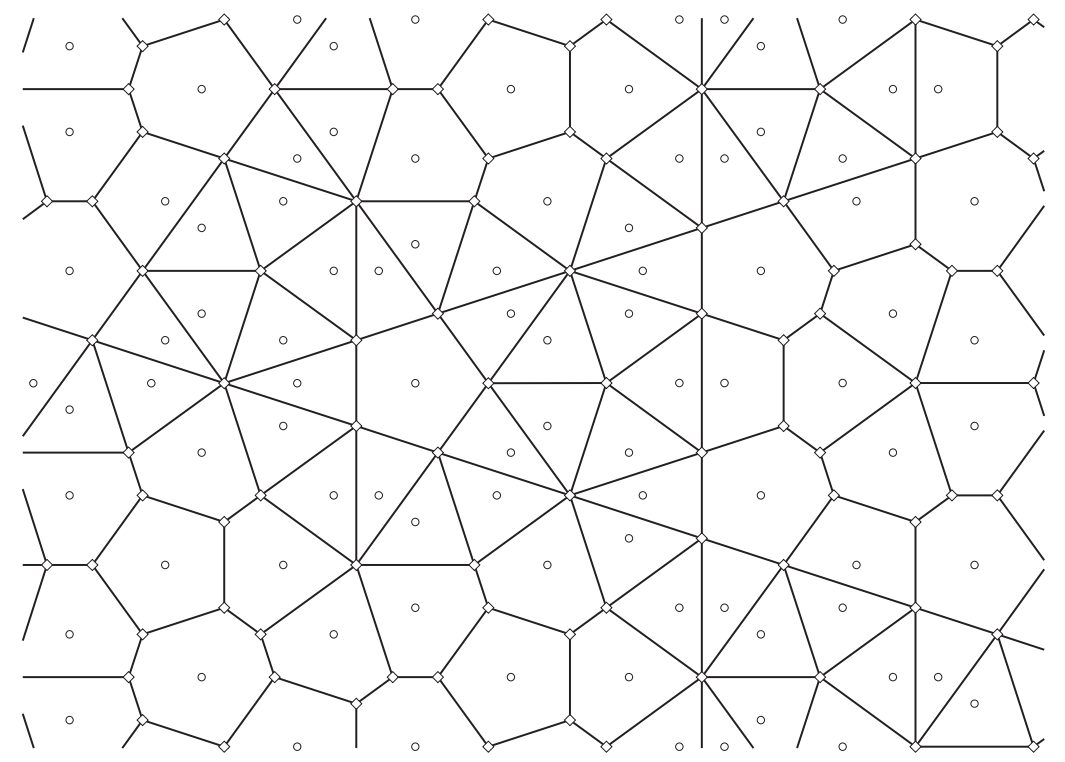

Fig. 5. Two Ising lattices on Penrose tiles. The spins on the odd sublattice (the first Ising model) are joined by bonds, but the spins on the even sublattice (the second Ising model on the dual lattice) are denoted by circles without their bonds. The two lattices are independent. The coordination number, which is the number of adjacent spins connected to a given spin in each sublattice, takes values $3,4,5,6$, or 7 . Incorporating the two models, a third Ising model can be defined with Ising spins on both the odd and even sublattices, i.e. the decoupling limit of Baxter's eight-vertex model on the Penrose lattice. $13 \mid 14]$ 15] 16 [17 
where $u_{j}^{\prime}=u_{j}$ for rapidity lines of type $j$ with arrows pointing to the same side of the line joining the two spins, and $u_{j}^{\prime}=u_{j} \pm \lambda$ for rapidities with arrows pointing to opposite sides of the line. It is as if the rapidities of lines pointing to the opposite side need to be flipped by adding $\pm \lambda$, i.e. adding $\pm \pi$ to the angle variable $u_{j} \pi / \lambda$. ${ }^{(48)}$ The functions $g$ have both the "permutation symmetry" (which means that they are invariant under all permutations of the rapidities) and the "difference property" (which implies a translation invariance under shifting all the rapidities by the same amount).(14)

We next examine in more detail when we have to choose $u_{j}^{\prime}=u_{j}$ or $u_{j}^{\prime}=u_{j} \pm \lambda$ in (3.1). Through a point in the mesh where spin $\sigma$ sits, as shown in Fig. 1, we draw five dashed lines parallel to each of the five grids. As the choice of the point in the mesh is rather arbitrary, the dashed lines should really have been drawn with a finite thickness, i.e. open strips without their boundaries between two consecutive grid lines of the pentagrid. Five arrows are also drawn perpendicular to the dashed lines to indicate the directions in which the integers $k_{j}$ increase. If the other spin $\sigma^{\prime}$ is in a mesh crossed by the $j$ th dashed line, i.e. $\sigma$ and $\sigma^{\prime}$ lie within the same strip, then the two spins have the same $K_{j}\left(\ell_{j}=0\right)$ and their pair correlation function does not depend on the value of $u_{j}^{\prime}$. When $\sigma^{\prime}$ moves away from this dashed line in the direction of the arrow, we have $\ell_{j} \geqslant 0$, whereas $\ell_{j} \leqslant 0$ if $\sigma^{\prime}$ moves away in the direction opposite to the arrow. These dashed lines (or more precisely strips) divide the entire plane into ten regions, and we numbered them from I to X. From Fig. 1, we can see that $\left(\ell_{0}, \cdots, \ell_{4}\right)$ have the same signs inside each region.

If $\sigma^{\prime}$ is in regions III or VIII, we find from Fig. 1 that the arrows of all the rapidity lines point to the same side of the line joining the two spins. Thus $u_{j}^{\prime}=u_{j}$ for all five $j$-values. When $\sigma^{\prime}$ is in regions II or VII, then the rapidity lines with $u_{4}$ and the other rapidity lines are pointing to opposite sides of the line joining the spins, so that $u_{4}^{\prime}=u_{4}-\lambda$ and $u_{j}^{\prime}=u_{j}$ for $j \neq 4$. For regions IV and IX the $u_{1}$ rapidity lines point in the other direction with respect to the other rapidity lines, implying $u_{1}^{\prime}=u_{1}+\lambda$. If $\sigma^{\prime}$ is in regions I and VI, the arrows of the $u_{2}$ and $u_{4}$ rapidity lines are on the opposite side and, therefore, $u_{4}^{\prime}=u_{4}-\lambda$ and $u_{2}^{\prime}=u_{2}-\lambda$. Similarly, for regimes $\mathrm{V}$ and $\mathrm{X}$, $u_{1}^{\prime}=u_{1}+\lambda$ and $u_{3}^{\prime}=u_{3}+\lambda$. For all other $j$-values, $u_{j}^{\prime}=u_{j}$. In summary, our choices for $u_{j}^{\prime}$ are listed in Table 【. with the $u_{j}$ 's given in (2.8), where we may set $u_{1}=0$ without loss of generality in view of the difference property of the pair correlation function. (14)

We may even shift the five rapidity values $u_{0}^{\prime}, \ldots, u_{4}^{\prime}$ in (3.1) by the same 
Table I. Rapidities

\begin{tabular}{|c|c|c|c|c|c|}
\hline Regions & $u_{4}^{\prime}$ & $u_{2}^{\prime}$ & $u_{0}^{\prime}$ & $u_{3}^{\prime}$ & $u_{1}^{\prime}$ \\
\hline I and VI & $u_{4}-\lambda$ & $u_{2}-\lambda$ & $u_{0}$ & $u_{3}$ & $u_{1}$ \\
\hline II and VII & $u_{4}-\lambda$ & $u_{2}$ & $u_{0}$ & $u_{3}$ & $u_{1}$ \\
\hline III and VIII & $u_{4}$ & $u_{2}$ & $u_{0}$ & $u_{3}$ & $u_{1}$ \\
\hline IV and IX & $u_{4}$ & $u_{2}$ & $u_{0}$ & $u_{3}$ & $u_{1}+\lambda$ \\
\hline V and X & $u_{4}$ & $u_{2}$ & $u_{0}$ & $u_{3}+\lambda$ & $u_{1}+\lambda$ \\
\hline
\end{tabular}

amount, depending on the choice of region, such that $\min _{j} u_{j}^{\prime}=0$. We can then also use the permutation property ${ }^{(14)}$ of the pair-correlation function $g$, given in (3.1), to rearrange the five resulting rapidity values $u_{j}^{\prime}$ in decreasing order as $\frac{4}{5} \lambda, \frac{3}{5} \lambda, \frac{2}{5} \lambda, \frac{1}{5} \lambda, 0$. Therefore, it suffices to calculate the quantity

$$
\begin{aligned}
& g\left[m_{4}, m_{3}, m_{2}, m_{1}, m_{0}\right] \equiv \\
& g(\overbrace{\frac{4 \lambda}{5}, \ldots, \frac{4 \lambda}{5}}^{m_{4}}, \overbrace{\frac{3 \lambda}{5}, \ldots, \frac{3 \lambda}{5}}^{m_{3}}, \overbrace{\frac{2 \lambda}{5}, \ldots, \frac{2 \lambda}{5}}^{m_{2}}, \overbrace{\frac{\lambda}{5}, \ldots, \frac{\lambda}{5}, 0, \ldots, 0}^{m_{1}} \overbrace{0}^{m_{0}}),
\end{aligned}
$$

where the $m_{j}$ 's are nonnegative integers depending on the $\ell_{j}$ 's and the choice of region. To determine this dependence, we can first use (2.8), from which we find $u_{3}+\lambda>u_{1}+\lambda>u_{4}>u_{2}>u_{0}>u_{3}>u_{1}>u_{4}-\lambda>u_{2}-\lambda$. Comparing (3.1), Table I and (2.8) we can then express all pair correlations in the form (3.2). We list the results for the ten different regions in Table II, This completes, more or less, the calculation of the pair correlation function, as we can refer to our previous papers ${ }^{(54] 60)}$ for further details on how to evaluate the $g\left[m_{4}, m_{3}, m_{2}, m_{1}, m_{0}\right]$ defined in (3.2).

Since the pentagrid is invariant under rotations by angles that are integer multiples of $2 \pi / 5$, the grids may be relabeled $m \rightarrow m+j,(\bmod 5)$. Then the differences of the five integer vectors of the two spins are also relabeled $\ell_{m} \rightarrow \ell_{m+j}$. This shows that the pair correlation function must have the cyclic property

$$
\left\langle\sigma \sigma^{\prime}\right\rangle_{\left[\ell_{0}, \ell_{1}, \ell_{2}, \ell_{3}, \ell_{4}\right]}=\left\langle\sigma \sigma^{\prime}\right\rangle_{\left[\ell_{j}, \ell_{j+1}, \ell_{j+2}, \ell_{j+3}, \ell_{j+4}\right]}, \quad(\bmod 5) .
$$


Table II. Pair-Correlation Function

\begin{tabular}{|c|c|c|}
\hline Regions & Signs of $\left(\ell_{0}, \ell_{1}, \ell_{2}, \ell_{3}, \ell_{4}\right)$ & $\left\langle\sigma \sigma^{\prime}\right\rangle\left[\ell_{0}, \cdots, \ell_{4}\right]=$ \\
\hline I \& VI & $(+,+,+,-,-) \&(-,-,-,+,+)$ & $g\left[\left|\ell_{0}\right|,\left|\ell_{3}\right|,\left|\ell_{1}\right|,\left|\ell_{4}\right|,\left|\ell_{2}\right|\right]$ \\
\hline II \& VII & $(+,+,-,-,-) \&(-,-,+,+,+)$ & $g\left[\left|\ell_{2}\right|,\left|\ell_{0}\right|,\left|\ell_{3}\right|,\left|\ell_{1}\right|,\left|\ell_{4}\right|\right]$ \\
\hline III \& VIII & $(+,+,-,-,+) \&(-,-,+,+,-)$ & $g\left[\left|\ell_{4}\right|,\left|\ell_{2}\right|,\left|\ell_{0}\right|,\left|\ell_{3}\right|,\left|\ell_{1}\right|\right]$ \\
\hline IV \& IX & $(+,-,-,-,+) \&(-,+,+,+,-)$ & $g\left[\left|\ell_{1}\right|,\left|\ell_{4}\right|,\left|\ell_{2}\right|,\left|\ell_{0}\right|,\left|\ell_{3}\right|\right]$ \\
\hline V \& X & $(+,-,-,+,+) \&(-,+,+,-,-)$ & $g\left[\left|\ell_{3}\right|,\left|\ell_{1}\right|,\left|\ell_{4}\right|,\left|\ell_{2}\right|,\left|\ell_{0}\right|\right]$ \\
\hline
\end{tabular}

From Table II, we indeed find that this property holds.

\section{Enumeration of sites}

As mentioned in the introduction in the context of the Fibonacci Ising model, ${ }^{(53[54)}$ to calculate the q-dependent susceptibility (1.1) for lattices for which the correlations are not translationally invariant, one needs to find a way to calculate suitable averages of the pair-correlation function. Since the meshes in the pentagrid - and even the distances between them - can be infinitesimally small in the thermodynamic limit, the problem of counting all the spin sites must be first solved.

We proceed by considering in detail the parallelograms bounded by two sets of parallel grid lines in the pentagrid and examining all possible spin sites in each of these parallelograms. Let $P\left(k_{j}, k_{j+1}\right)$ denote the parallelogram sandwiched between four grid lines $k_{j}-1, k_{j}, k_{j+1}-1$ and $k_{j+1}$ for any $j$. (Throughout the entire paper, we let $k_{j+5} \equiv k_{j}$, i.e. the index $j$ is considered mod 5.) Obviously, for all points $z \in P\left(k_{j}, k_{j+1}\right)$, we have $K_{j}(z)=k_{j}$ and $K_{j+1}(z)=k_{j+1}$. The different choices of $j$ give the different orientations of the parallelograms. Next, we determine how many spin sites a parallelogram may have, what are the integer vectors for these spins, etc. Since this section is rather lengthy, we have subdivided it into many parts, and put the main conclusion at the end. 


\subsection{Reference vector for $P$}

The vertices of parallelogram $P\left(k_{j}, k_{j+1}\right)$ can be calculated from (2.3) as the intersections of grid lines in grids $G_{j}$ and $G_{j+1}$, i.e.

$$
G_{j} \cap G_{j+1}=\left\{z \in \mathbb{C} \mid z=\frac{\mathrm{i}\left[\zeta^{j}\left(k_{j+1}-\gamma_{j+1}\right)-\zeta^{j+1}\left(k_{j}-\gamma_{j}\right)\right]}{\sin (2 \pi / 5)}\right\},
$$

for $k_{j}, k_{j+1} \in \mathbb{Z}$. Moreover, any point $z$ in the interior of $P\left(k_{j}, k_{j+1}\right)$ may be expressed in terms of $\boldsymbol{\epsilon}=\left(\epsilon_{j}, \epsilon_{j+1}\right)$, with $0 \leqslant \epsilon_{j}, \epsilon_{j+1} \leqslant 1$, as

$$
z=\frac{\mathrm{i}\left[\zeta^{j}\left(k_{j+1}-\gamma_{j+1}-\epsilon_{j+1}\right)-\zeta^{j+1}\left(k_{j}-\gamma_{j}-\epsilon_{j}\right)\right]}{\sin (2 \pi / 5)} \equiv z(\boldsymbol{\epsilon}),
$$

allowing us a change of notation $K_{j+\mathrm{m}}(\boldsymbol{\epsilon}) \equiv K_{j+\mathrm{m}}(z(\boldsymbol{\epsilon}))$ for $z \in P$.

The four corners of parallelogram $P\left(k_{j}, k_{j+1}\right)$ are given by $\boldsymbol{\epsilon}=(0,0),(0,1)$, $(1,0)$, or $(1,1)$ as can be seen from (4.1). Now for each $P\left(k_{j}, k_{j+1}\right)$, we pick a reference integer vector $\left(k_{0}, \cdots, k_{4}\right)$, which is related to the integer vector of the corner of $P\left(k_{j}, k_{j+1}\right)$ with $\boldsymbol{\epsilon}=(0,0)$. Apart from the obvious identities $k_{j}=K_{j}(\mathbf{0})$ and $k_{j+1}=K_{j+1}(\mathbf{0})$, we have

$$
\begin{aligned}
k_{j+2} & =K_{j+2}(\mathbf{0}) \\
& =\left\lceil p^{-1}\left(k_{j+1}-\gamma_{j+1}\right)-k_{j}+\gamma_{j}+\gamma_{j+2}\right\rceil=\lceil\alpha\rceil-k_{j}, \\
k_{j+4} & =K_{j+4}(\mathbf{0}) \\
& =\left\lceil p^{-1}\left(k_{j}-\gamma_{j}\right)-k_{j+1}+\gamma_{j+1}+\gamma_{j+4}\right\rceil=\lceil\beta\rceil-k_{j+1},
\end{aligned}
$$

in which

$$
\begin{aligned}
& \alpha \equiv \hat{\alpha}\left(k_{j+1}\right) \equiv p^{-1}\left(k_{j+1}-\gamma_{j+1}\right)+\gamma_{j}+\gamma_{j+2}, \\
& \beta \equiv \hat{\beta}\left(k_{j}\right) \equiv p^{-1}\left(k_{j}-\gamma_{j}\right)+\gamma_{j+1}+\gamma_{j+4} .
\end{aligned}
$$

However, for the last component of the reference integer vector we choose

$$
k_{j+3}=2-\lceil\alpha\rceil-\lceil\beta\rceil=-\lfloor\alpha\rfloor-\lfloor\beta\rfloor \not \equiv K_{j+3}(\mathbf{0}),
$$

where $\lfloor x\rfloor$ denotes the "floor of $x$ ", which is the largest integer $\leqslant x$ and $\lfloor x\rfloor=\lceil x\rceil$ if and only if $x \in \mathbb{Z}$. Since the pentagrid is regular, we find $\alpha, \beta \notin \mathbb{Z}$ and the second equality in the above equation holds. The index of 
any mesh, whose integer vector is $\vec{K}(z)$, is defined as $\sum_{j} K_{j}(z)$. It is shown by de Bruijn (5) that it has one of the four possible values, $1,2,3$, or 4 . We associate odd spins to meshes with index 1 or 3 , and even spins to meshes with index 2 or 4 . The index of the reference integer vector is $\sum_{j} k_{j}=2$.

From (2.4) and (4.2) we find

$$
\left.K_{j+3}(\mathbf{0})=\left\lceil-p^{-1}\left(k_{j+1}-\gamma_{j+1}+k_{j}-\gamma_{j}\right)+\gamma_{j+3}\right)\right\rceil=\lceil-\alpha-\beta\rceil .
$$

Using

$$
\{x\}=x-\lfloor x\rfloor, \quad\lceil-x-y\rceil=-\lfloor x\rfloor-\lfloor y\rfloor+\lceil-\{x\}-\{y\}\rceil
$$

and comparing (4.5) with (4.6), we find

$$
K_{j+3}(\mathbf{0})=\left\{\begin{array}{lll}
k_{j+3}-1 & \text { for } & \{\alpha\}+\{\beta\} \geqslant 1 \\
k_{j+3} & \text { for } & \{\alpha\}+\{\beta\}<1 .
\end{array}\right.
$$

This shows the mesh below the upper right corner with $\boldsymbol{\epsilon}=(0,0)$ belongs to the even sublattice and its integer vector is the reference vector of $P$ only for $\{\alpha\}+\{\beta\}<1$, but not for $\{\alpha\}+\{\beta\} \geqslant 1$.

It would be more natural to choose this corner as our reference and to compare the integer vectors of other spins inside $P \equiv P\left(k_{j}, k_{j+1}\right)$ with it. This was what we did originally. However, we find that the rather odd choice of the reference vector given by (4.3) and (4.5), which may not even be the integer vector of a mesh, has made calculations much simpler. We next examine the differences between the integer vectors of the other spins in $P\left(k_{j}, k_{j+1}\right)$ with respect to this reference vector.

\subsection{Integer vectors for $z \in P$}

Substituting (4.2) into (2.4) and using (4.3) and (4.5), we evaluate the integer vectors $\vec{K}(\boldsymbol{\epsilon})$ for every point in $P$ that is not on a grid line of the pentagrid. We find that its components for $\mathrm{m}=2,3,4$ are given by

$$
\begin{aligned}
& K_{j+\mathrm{m}}(\boldsymbol{\epsilon})=k_{j+\mathrm{m}}+\partial K_{j+\mathrm{m}}(\boldsymbol{\epsilon}), \\
& \partial K_{j+\mathrm{m}}(\boldsymbol{\epsilon})=\left\lceil\lambda_{j+\mathrm{m}}(\boldsymbol{\epsilon})-1\right\rceil=\left\lfloor\lambda_{j+\mathrm{m}}(\boldsymbol{\epsilon})\right\rfloor,
\end{aligned}
$$

with

$$
\lambda_{j+2}(\boldsymbol{\epsilon})=\{\alpha\}+\epsilon_{j}-p^{-1} \epsilon_{j+1}, \quad \lambda_{j+4}(\boldsymbol{\epsilon})=\{\beta\}+\epsilon_{j+1}-p^{-1} \epsilon_{j} .
$$


and

$$
\lambda_{j+3}(\boldsymbol{\epsilon})=p^{-1}\left(\epsilon_{j}+\epsilon_{j+1}\right)-\{\alpha\}-\{\beta\}+1 .
$$

The last equality in (4.9) does not hold if $\lambda_{j+\mathrm{m}}(\boldsymbol{\epsilon})$ is an integer, i.e. if the point is on a grid line of grid $G_{j+\mathrm{m}}$. If we define the difference vector $\partial \vec{K}(\boldsymbol{\epsilon})$ for each mesh in $P$ as 7

$$
\partial \vec{K}(\boldsymbol{\epsilon})=\left[\partial K_{j+2}(\boldsymbol{\epsilon}), \partial K_{j+3}(\boldsymbol{\epsilon}), \partial K_{j+4}(\boldsymbol{\epsilon})\right]
$$

then for $\{\alpha\}+\{\beta\}>1$, we have $\partial \vec{K}(\mathbf{0})=[0,-1,0]$, and for $\{\alpha\}+\{\beta\}<1$, $\partial \vec{K}(\mathbf{0})=[0,0,0]$.

For fixed $\gamma_{j}$ 's with $j=0, \cdots, 4$, which are the shifts of the pentagrid, the $\hat{\alpha}\left(k_{j+1}\right)$ and $\hat{\beta}\left(k_{j}\right)$ in (4.4) are uniquely determined for each $P\left(k_{j}, k_{j+1}\right)$. Consequently, the number of meshes in $P$ and the difference vectors $\partial \vec{K}$ for each mesh are uniquely determined by (4.9) to (4.11). The configurations of two parallelograms $P$ and $P^{\prime}$ are the same, if they have the same number of meshes (spin sites), and the same sets of difference vectors. The difference in the configurations does not depend on the exact locations of the relevant grid lines or their intersections. However, whenever a grid line or an intersection moves in or out of the parallelogram $P\left(k_{j}, k_{j+1}\right)$, the configuration changes.

\subsection{Relevant grid lines}

It is easy to see from (2.4) that $\partial K_{j+\mathrm{m}}(\boldsymbol{\epsilon})$ changes its value whenever lines in the $(j+\mathrm{m})$ th grid are crossed. Because $0 \leqslant\{x\}, \epsilon_{j}, \epsilon_{j+1}<1$, we find from (4.10) and (4.11) that

$$
-1<\lambda_{j+2}(\boldsymbol{\epsilon}), \lambda_{j+4}(\boldsymbol{\epsilon})<2, \quad-1<\lambda_{j+3}(\boldsymbol{\epsilon})<3 .
$$

Consequently, the only relevant grid lines for the parallelogram $P\left(k_{j}, k_{j+1}\right)$ are those having integer labels $k_{j+2}-1+n^{\prime}, k_{j+3}-1+m$, or $k_{j+4}-1+n$, with $n, n^{\prime}=0,1$ and $m=0,1,2$. Indeed, these are the only integer values that the $K_{j+\mathrm{m}}(\boldsymbol{\epsilon})$ in (4.9) , or $k_{j+\mathrm{m}}-1+\left\lceil\lambda_{j+\mathrm{m}}(\boldsymbol{\epsilon})\right\rceil$, can assume. The loci of these lines are given by linear equations in $\epsilon_{j}$ and $\epsilon_{j+1}$ as

$$
\lambda_{j+2}(\boldsymbol{\epsilon})=n^{\prime}, \quad \lambda_{j+3}(\boldsymbol{\epsilon})=m, \quad \lambda_{j+4}(\boldsymbol{\epsilon})=n .
$$

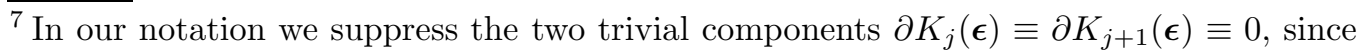
in parallelogram $P$ we have by definition $K_{j}(\boldsymbol{\epsilon}) \equiv k_{j}, K_{j+1}(\boldsymbol{\epsilon}) \equiv k_{j+1}$.
} 
From (4.10), we find

$$
\begin{array}{lll}
0<\lambda_{j+2}(\boldsymbol{\epsilon})<2 & \text { if } & \{\alpha\}>p^{-1}, \\
0<\lambda_{j+4}(\boldsymbol{\epsilon})<2 & \text { if } & \{\beta\}>p^{-1} .
\end{array}
$$

Therefore the equation $\lambda_{j+2}=0\left(\lambda_{j+4}=0\right)$ cannot be satisfied if $\{\alpha\}>p^{-1}$ $\left(\{\beta\}>p^{-1}\right)$, while equations $\lambda_{j+2}=1$ and $\lambda_{j+4}=1$ always have solutions in $P$. This means that the only grid lines in grids $G_{j+2}$ and $G_{j+4}$ crossing $P$ are given by

$$
\begin{array}{ll}
k_{j+2}, k_{j+4} \in P & \text { always, } \\
k_{j+2}-1 \in P & \text { if } \quad\{\alpha\}<p^{-1} \\
k_{j+4}-1 \in P & \text { if } \quad\{\beta\}<p^{-1}
\end{array}
$$

From (4.11) we find that the grid lines $k_{j+3}+m$ are parallel to the diagonal $\epsilon_{j}+\epsilon_{j+1}=1$, and

$$
\begin{array}{cc}
1-p^{-3}<\lambda_{j+3}(\boldsymbol{\epsilon})<2 p^{-1}+1 & \text { if } \quad 0<\{\alpha\}+\{\beta\}<p^{-3}, \\
0<\lambda_{j+3}(\boldsymbol{\epsilon})<2 & \text { if } \quad p^{-3}<\{\alpha\}+\{\beta\}<1, \\
-p^{-3}<\lambda_{j+3}(\boldsymbol{\epsilon})<2 p^{-1} & \text { if } 1<\{\alpha\}+\{\beta\}<2 p^{-1}, \\
-1<\lambda_{j+3}(\boldsymbol{\epsilon})<1 & \text { if } \quad 2 p^{-1}<\{\alpha\}+\{\beta\}<2,
\end{array}
$$

where we used the identity $p^{-3}=2 p^{-1}-1$. This means, there can be at most two lines of grid $G_{j+3}$ going through the inside of the parallelogram. We find that $\lambda_{j+3}=2$ has a solution in $P$ if $0<\{\alpha\}+\{\beta\}<p^{-3} ; \lambda_{j+3}=1$ has a solution in $P$ for $0<\{\alpha\}+\{\beta\}<2 p^{-1} ; \lambda_{j+3}=0$ has a solution in $P$ for $1<\{\alpha\}+\{\beta\}$. These facts can be summarized as

$$
\begin{array}{ll}
k_{j+3}+1 \in P & \text { if } \quad\{\alpha\}+\{\beta\}<p^{-3}=\sqrt{5}-2, \\
k_{j+3} \in P & \text { if } \quad\{\alpha\}+\{\beta\}<2 p^{-1}=\sqrt{5}-1, \\
k_{j+3}-1 \in P & \text { if } \quad\{\alpha\}+\{\beta\}>1 .
\end{array}
$$

At this point one may note the symmetry under $k_{j+2} \leftrightarrow k_{j+4}$ and $\alpha \leftrightarrow \beta$ in conditions (4.16) and (4.18). 


\subsection{Intersections of the grid lines}

Next, we need to calculate the positions of the intersections. We let $\boldsymbol{a}^{n, m}$ denote the intersection of a pair of grid lines in $G_{j+3}$ and $G_{j+4}$ numbered $k_{j+3}-1+m$ and $k_{j+4}-1+n, \boldsymbol{b}^{n^{\prime}, m}$ the intersection of lines $k_{j+3}-1+m$ and $k_{j+2}-1+n^{\prime}$ in $G_{j+3}$ and $G_{j+2}$, while $\boldsymbol{c}^{n, n^{\prime}}$ the intersection of lines $k_{j+4}-1+n$ and $k_{j+2}-1+n^{\prime}$ in $G_{j+4}$ and $G_{j+2}$. The locations of these intersections are found by solving the corresponding linear equations given in (4.14). We find

$$
\begin{aligned}
& \boldsymbol{a}^{n, m}=\left(p(\{\beta\}-n)+m+n-1+\{\alpha\}, p^{-1}(\{\alpha\}+n+m-1)\right), \\
& \boldsymbol{b}^{n^{\prime}, m}=\left(p^{-1}\left(\{\beta\}+m+n^{\prime}-1\right), p\left(\{\alpha\}-n^{\prime}\right)+m+n^{\prime}-1+\{\beta\}\right), \\
& \boldsymbol{c}^{n, n^{\prime}}=\left(p n^{\prime}+n-p\{\alpha\}-\{\beta\}, p n+n^{\prime}-\{\alpha\}-p\{\beta\}\right) .
\end{aligned}
$$

Clearly, whenever both components $\left(\epsilon_{j}, \epsilon_{j+1}\right)$ of an intersection are positive and less than 1 , it is inside the parallelogram $P\left(k_{j}, k_{j+1}\right)$. This way, we find from (4.19) the conditions for the three possible cases, namely

$$
\begin{array}{lll}
\boldsymbol{a}^{0,1} \in P\left(k_{j}, k_{j+1}\right) & \text { if } \quad\{\beta\}<p^{-1}-p^{-1}\{\alpha\}, \\
\boldsymbol{a}^{1,0} \in P\left(k_{j}, k_{j+1}\right) & \text { if } \quad\{\beta\}>1-p^{-1}\{\alpha\} \\
\boldsymbol{a}^{1,1} \in P\left(k_{j}, k_{j+1}\right) & \text { if } \quad\left\{\begin{array}{l}
0<\{\alpha\}<p^{-1} \text { and } \\
p^{-2}-p^{-1}\{\alpha\}<\{\beta\}<1-p^{-1}\{\alpha\} .
\end{array}\right.
\end{array}
$$

Similarly, from (4.20) we obtain

$$
\begin{array}{ll}
\boldsymbol{b}^{0,1} \in P\left(k_{j}, k_{j+1}\right) \quad \text { if } \quad\{\beta\}<1-p\{\alpha\}, & \\
\boldsymbol{b}^{1,0} \in P\left(k_{j}, k_{j+1}\right) & \text { if } \quad\{\beta\}>p(1-\{\alpha\}), \\
\boldsymbol{b}^{1,1} \in P\left(k_{j}, k_{j+1}\right) & \text { if } \quad\left\{\begin{array}{l}
0<\{\beta\}<p^{-1} \text { and } \\
p^{-2}-p^{-1}\{\beta\}<\{\alpha\}<1-p^{-1}\{\beta\},
\end{array}\right.
\end{array}
$$

and from (4.21) we get

$$
\begin{array}{ll}
\boldsymbol{c}^{0,1} \in P\left(k_{j}, k_{j+1}\right) & \text { if } \quad p^{-1}-p\{\alpha\}<\{\beta\}<p^{-1}(1-\{\alpha\}), \\
\boldsymbol{c}^{1,0} \in P\left(k_{j}, k_{j+1}\right) & \text { if } \quad p^{-2}-p^{-1}\{\alpha\}<\{\beta\}<1-p\{\alpha\}, \\
\boldsymbol{c}^{1,1} \in P\left(k_{j}, k_{j+1}\right) & \text { if } \quad\{\beta\}>\max \left(1-p^{-1}\{\alpha\}, p-p\{\alpha\}\right) .
\end{array}
$$


Note the symmetry between (4.22) and (4.23) and between the first two lines of (4.24) under $\alpha \leftrightarrow \beta$ and implicitly $k_{j+2} \leftrightarrow k_{j+4}$.

Three lines cannot exactly meet in a common intersection, as was shown by de Bruijn ${ }^{(5)}$ for a regular pentagrid. However, they can meet arbitrarily close and the theoretical limiting conditions of triple intersection

$$
\begin{array}{lll}
\text { "a } \boldsymbol{a}^{1,0}=\boldsymbol{b}^{1,0}=\boldsymbol{c}^{1,1} " & \Longleftrightarrow & \{\alpha\}+\{\beta\}=p, \\
\text { " } \boldsymbol{a}^{0,1}=\boldsymbol{b}^{1,1}=\boldsymbol{c}^{0,1} " & \Longleftrightarrow & \{\alpha\}+\{\beta\}=p^{-1}, \\
\text { " } \boldsymbol{a}^{1,1}=\boldsymbol{b}^{0,1}=\boldsymbol{c}^{1,0 "} \quad \Longleftrightarrow \quad\{\alpha\}+\{\beta\}=p^{-1},
\end{array}
$$

play a role in the following subsection.

\subsection{The twenty-four allowed configurations}

Next, we use (4.16), (4.18), (4.22)-(4.25) to study how the configuration $C(m)$ of parallelogram $P\left(k_{j}, k_{j+1}\right)$ depends on the values of $\{\alpha\}=\left\{\hat{\alpha}\left(k_{j+1}\right)\right\}$ and $\{\beta\}=\left\{\hat{\beta}\left(k_{j}\right)\right\}$. We show the various cases in Fig. 6 for $j=0$. For $j \neq 0$ we need to rotate each picture $j$ times $72^{\circ}$.

For $\{\alpha\},\{\beta\}>p^{-1}$, the three grid lines $k_{j+2}, k_{j+3}-1, k_{j+4}$ and their three intersections $\boldsymbol{a}^{1,0}, \boldsymbol{b}^{1,0}, \boldsymbol{c}^{1,1}$ are inside the parallelogram, producing seven meshes in $P\left(k_{j}, k_{j+1}\right)$, as shown in Figs. 6(a) and 6(b). The difference between the two cases is that the intersection $\boldsymbol{c}^{1,1}$ is on opposite sides of the grid line $k_{j+3}-1$, as the sign of $\{\alpha\}+\{\beta\}-p$ changes, cf. (4.25). The grid line $k_{j+3}-1$ moves upward toward the upper right corner as $\{\beta\}$ decreases. It is below the diagonal $\epsilon_{j}+\epsilon_{j+1}=1$ for $\{\alpha\}+\{\beta\}>p$, corresponding to Fig. 6 (a), and above the diagonal for $\{\alpha\}+\{\beta\}<p$, as shown in Fig. [6(b). The index of the inner triangle changes from odd to even in view of (4.10), (4.11) and (4.14). Hence, the spin configurations for the two cases are different: $\mathrm{C}(1)$ in Fig. 6(a) has 4 odd sites and 3 even sites, and $C(2)$ shown in Fig. 6)(b) has 3 odd sites and 4 even sites. Also, $\mathrm{C}(1)$ is the only configuration for which the reference integer vector does not correspond to an actual mesh.

For $\{\alpha\}>p^{-1}$ and $0<\{\beta\}<p^{-1}$, we have six cases denoted by $\mathrm{C}(3)$ to $\mathrm{C}(8)$ arranged in the decreasing order of $\{\beta\}$. For $1-p^{-1}\{\alpha\}<\{\beta\}$, the configuration $\mathrm{C}(3)$ is almost the same as $\mathrm{C}(2)$ except having one more even site because line $k_{j+4}-1$ is now inside the parallelogram, as shown in Fig. 6(c). As $\{\beta\}$ decreases, line $k_{j+4}$ moves downward and for $2 p^{-1}-\{\alpha\}<$ $\{\beta\}<1-p^{-1}\{\alpha\}$, the intersections of line $k_{j+4}$ with lines $k_{j+3}-1\left(\boldsymbol{a}^{1,0}\right)$ and 


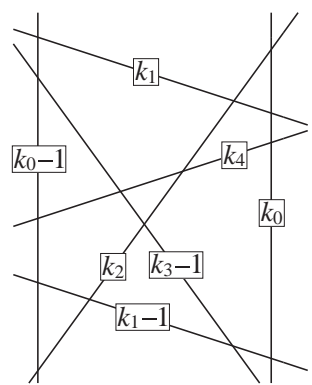

(a) $\mathrm{C}(1): \quad N=7$ $N_{\mathrm{O}}=4, N_{\mathrm{e}}=3$

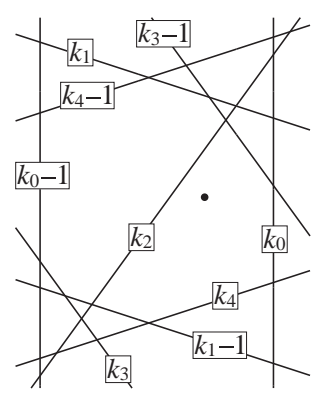

(e) $\begin{aligned} & \mathrm{C}(5): N=7 \\ & N_{\mathrm{O}}=3, N_{\mathrm{e}}=\end{aligned}$

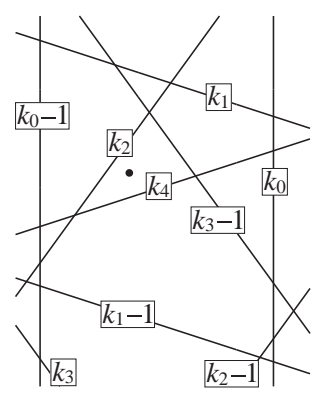

(i) $\mathrm{C}(9): N=8$

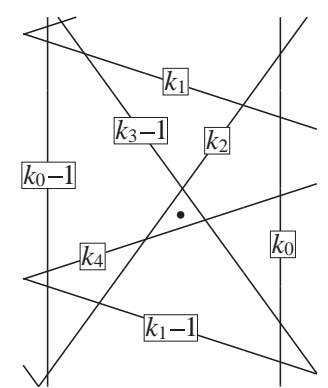

(b) $\begin{aligned} & \mathrm{C}(2): N=7 \\ & N_{\mathrm{O}}=3, N_{\mathrm{e}}=4\end{aligned}$

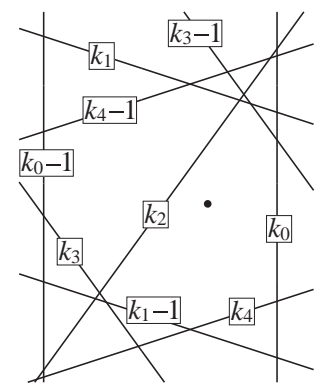

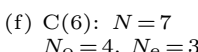

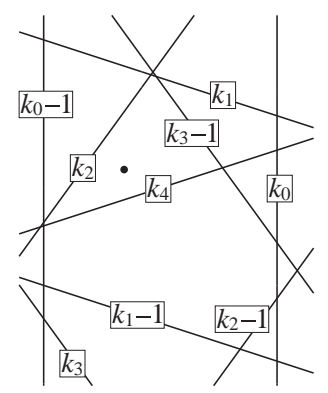

(j) $\mathrm{C}(10): \quad N=6$

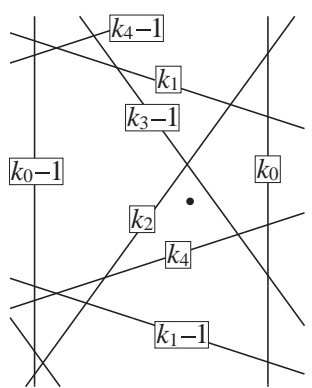

(c) $\begin{aligned} & \mathrm{C}(3): N=8 \\ & N_{\mathrm{O}}=3, N_{\mathrm{e}}=5\end{aligned}$

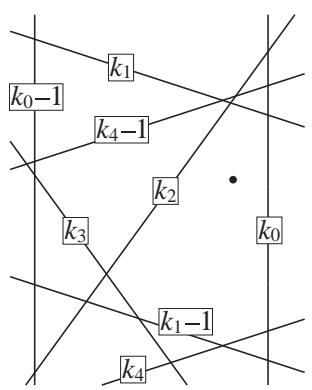

(g) $\begin{aligned} & \mathrm{C}(7): N=6 \\ & N_{\mathrm{o}}=3, N_{\mathrm{e}}=3\end{aligned}$

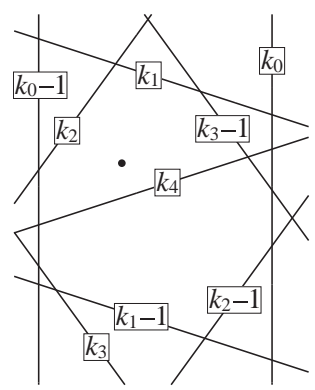

(k) C(11): $N=7$

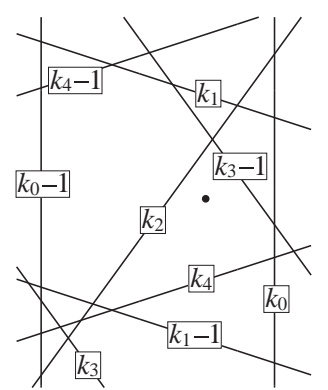

(d) $\begin{aligned} & \mathrm{C}(4): N=6 \\ & N_{\mathrm{O}}=3, N_{\mathrm{e}}=3\end{aligned}$

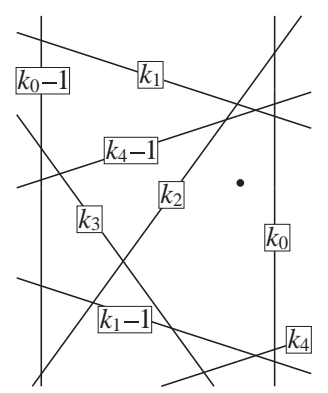

(h) $\begin{aligned} & \mathrm{C}(8): N=8 \\ & N_{\mathrm{O}}=5, N_{\mathrm{e}}=3\end{aligned}$

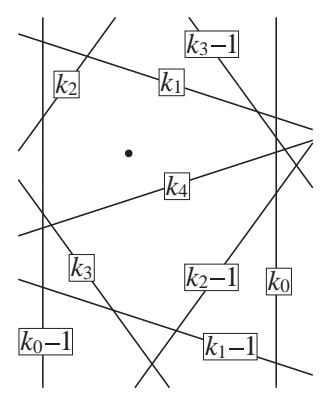

(1) $\mathrm{C}(12): \quad N=7$

Fig. 6. The spin configurations $\mathrm{C}(m), m=1, \ldots, 24$, for parallelogram $P\left(k_{0}, k_{1}\right)$ are shown in (a) through (x), where $N$ denotes the total number of spin sites inside $P\left(k_{0}, k_{1}\right)$, $N_{\mathrm{o}}$ the number of odd spins sites, and $N_{\mathrm{e}}$ the number of even spin sites. The mesh that contains the dot is the special even site whose integer vector is the reference integer vector for $P\left(k_{0}, k_{1}\right)$, such that $\partial \vec{K}=(0,0,0)$. For $\{\alpha\}+\{\beta\}>p$, such a site does not exist, as seen in 6(a). For $\{\alpha\}+\{\beta\}<1$, this site is the mesh right below the upper right corner of $P$. 


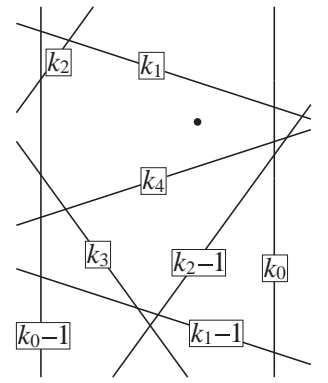

(m) $\mathrm{C}(13): N=6$

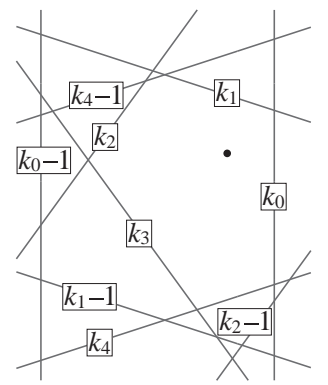

(q) C(17): $N=10$

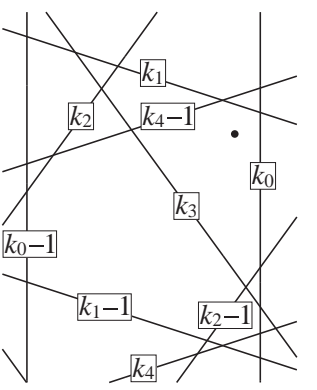

(u) C(21): $N=10$ $N_{\mathrm{o}}=5, N_{\mathrm{e}}=5$

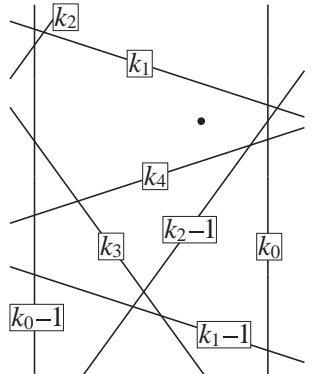

(n) $\begin{array}{ll}\mathrm{C}(14): & N=8 \\ N_{\mathrm{o}}=5, & N_{\mathrm{e}}=3\end{array}$

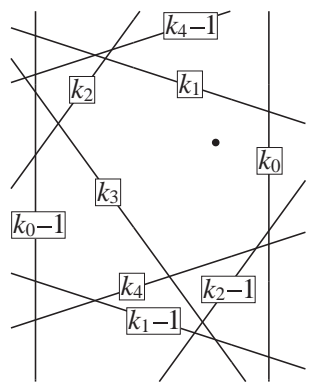

(r) C(18): $N=10$

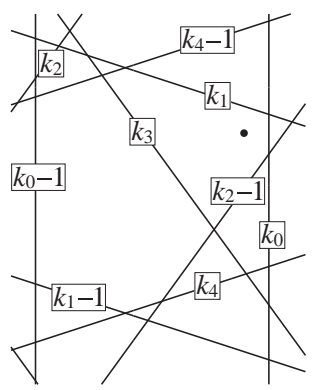

(v) $\mathrm{C}(22): N=10$ $N_{\mathrm{O}}=5, N_{\mathrm{e}}=5$

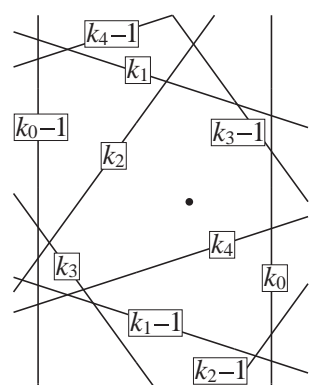

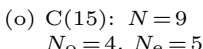

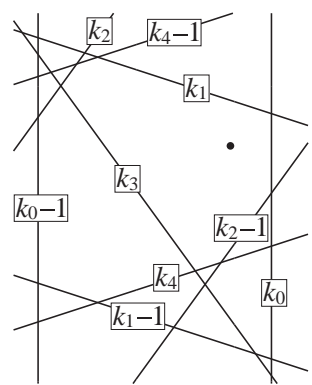

(s) $\mathrm{C}(19): \quad N=12$

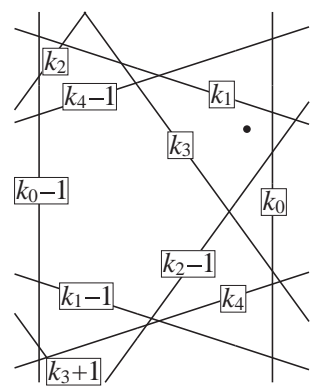

(w) $\mathrm{C}(23): N=8$ $N_{\mathrm{o}}=5, N_{\mathrm{e}}=3$

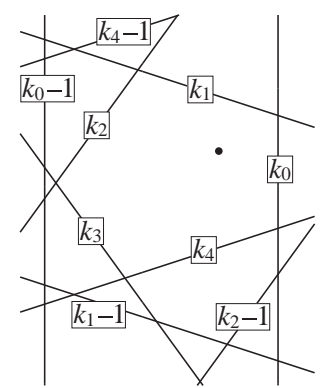

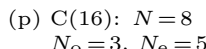

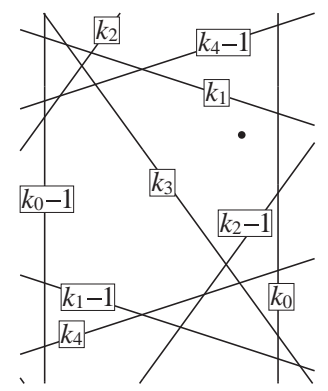

(t) $\mathrm{C}(20): N=12$

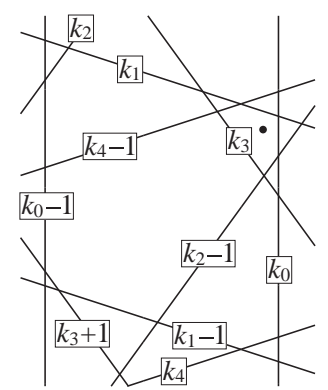

(x) $\mathrm{C}(24): N=9$

Fig. 6. Continued: The remaining twelve configurations. The number of spin sites $N$ varies between 6 and 12, whereas $N_{\mathrm{o}}$ and $N_{\mathrm{e}}$ vary between 3 and 7 . 
$k_{j+2}\left(\boldsymbol{c}^{1,1}\right)$ are seen from (4.22) and (4.24) to be outside of $P\left(k_{j}, k_{j+1}\right)$. As a result, two of the even sites are now outside, and $\mathrm{C}(4)$, shown in Fig. [6(d), has only six sites, three of which are odd, and three even.

As $\{\beta\}$ decreases further to $p(1-\{\alpha\})<\{\beta\}<2 p^{-1}-\{\alpha\}$, line $k_{j+3}$ is now inside $P\left(k_{j}, k_{j+1}\right)$, as seen from (4.18). Thus, configuration $\mathrm{C}(5)$, shown in Fig. 6(e), has one more even site than $\mathrm{C}(4)$. Both lines $k_{j+3}$ and $k_{j+3}-1$ move upward as $\{\beta\}$ decreases. For $1-\{\alpha\}<\{\beta\}<p(1-\{\alpha\})$, the intersection $\boldsymbol{b}^{1,1}$ of line $k_{j+3}$ with $k_{j+2}$ moves inside of $P\left(k_{j}, k_{j+1}\right)$, while $\boldsymbol{b}^{1,0}$, which is the intersection of $k_{j+3}-1$ and $k_{j+2}$, moves out, giving rise to configuration $\mathrm{C}(6)$ shown in Fig. 6](f), with three even sites and four odd sites.

For $p^{-1}(1-\{\alpha\})<\{\beta\}<1-\{\alpha\}$, line $k_{j+3}-1$ moves out of $P$, as shown in Fig. 6(g). Its configuration $\mathrm{C}(7)$ has 3 odd sites and 3 even sites differing from $\mathrm{C}(6)$ in that the odd site with $\partial \vec{K}(\mathbf{0})=(0,-1,0)$ is now outside $P\left(k_{j}, k_{j+1}\right)$. For $0<\{\beta\}<p^{-1}(1-\{\alpha\})$, the intersections $\boldsymbol{a}^{0,1}$ and $\boldsymbol{c}^{0,1}$ of line $k_{j+4}-1$ with $k_{j+3}$ and $k_{j+2}$ are now seen from (4.22) and (4.24) to be inside of $P\left(k_{j}, k_{j+1}\right)$ adding 2 more odd sites to $\mathrm{C}(8)$, which is shown in Fig. 6 (h).

In Fig. 6](i) through Fig. [6(n), the six cases $\mathrm{C}(9)$ through $\mathrm{C}(14)$ are shown for $\{\alpha\}<p^{-1}$ and $\{\beta\}>p^{-1}$. Because of (4.3) and (4.4) we can use the reflection symmetry $\{\alpha\} \leftrightarrow\{\beta\}, k_{j} \leftrightarrow k_{j+1}, k_{j+2} \leftrightarrow k_{j+4}$, which was noted also in the previous subsections. Thus these cases are similar to the configurations $\mathrm{C}(3)$ to $\mathrm{C}(8)$, and obtainable simply by replacing $k_{j+4}+n$ by $k_{j+2}+n$ and vice versa. To summarize, we find $\mathrm{C}(9)$ has 3 odd sites and 5 even sites; C(10) 3 odd sites and 3 even sites; C(11) 3 odd sites and 4 even sites; C(12) 4 odd sites and 3 even sites; C(13) has 3 odd sites and 3 even sites, and $\mathrm{C}(14) 5$ odd sites and 3 even sites.

For $\{\alpha\},\{\beta\}<p^{-1}$, at least five grid lines $k_{j+2}, k_{j+2}-1, k_{j+3}, k_{j+4}$ and $k_{j+4}-1$ are inside $P\left(k_{j}, k_{j+1}\right)$. In Fig. 6(o), we show configuration $\mathrm{C}(15)$ valid for $1<\{\alpha\}+\{\beta\}$, when both lines $k_{j+3}-1$ and $k_{j+3}$ and the intersections $\boldsymbol{b}^{1,1}$ and $\boldsymbol{a}^{1,1}$ are inside $P\left(k_{j}, k_{j+1}\right)$, as seen from (4.22) and (4.23). Configuration $\mathrm{C}(15)$ has 4 odd sites and 5 even sites.

For the region satisfying the three inequalities $\{\beta\}<1-\{\alpha\},\{\beta\}>$ $1-p\{\alpha\}$ and $\{\beta\}>p^{-1}(1-\{\alpha\})$, grid line $k_{j+3}$ and the corresponding odd site with $\partial \vec{K}=(0,-1,0)$ are now outside $P\left(k_{j}, k_{j+1}\right)$, such that configuration $\mathrm{C}(16)$ shown in Fig. 6(p) has one site less than $\mathrm{C}(15)$. It has 3 odd and 5 even sites.

For $1-p\{\alpha\}<\{\beta\}<p^{-1}(1-\{\alpha\})$, the intersections $\boldsymbol{a}^{0,1}$ and $\boldsymbol{c}^{0,1}$ of line $k_{j+4}-1$ are both also inside $P\left(k_{j}, k_{j+1}\right)$, as seen from (4.22) and (4.24), 
adding two odd sites to $\mathrm{C}(16)$. Thus, $\mathrm{C}(17)$ in Fig. 6(q) has 5 odd sites and 5 even. However, for $p^{-1}(1-\{\alpha\})<\{\beta\}<1-p\{\alpha\}$ the intersections $\boldsymbol{b}^{0,1}$ and $\boldsymbol{c}^{1,0}$ of lines $k_{j+2}-1$ are now inside instead, adding two different odd sites to configuration $\mathrm{C}(16)$. The resulting configuration $\mathrm{C}(18)$ shown in Fig. 6)(r) also has ten sites and relates to $\mathrm{C}(17)$ by the above reflection symmetry.

When both conditions $p^{-1}-p\{\alpha\}<\{\beta\}<1-p\{\alpha\}$ and $p^{-2}-p^{-1}\{\alpha\}<$ $\{\beta\}<p^{-1}(1-\{\alpha\})$ are satisfied, we find six intersections $\boldsymbol{a}^{1,1}, \boldsymbol{b}^{1,1}, \boldsymbol{a}^{0,1}, \boldsymbol{b}^{0,1}$, $c^{0,1}$ and $\boldsymbol{c}^{1,0}$ inside $P\left(k_{j}, k_{j+1}\right)$. As a result, there are 12 sites for the two cases $\mathrm{C}(19)$ and $\mathrm{C}(20)$ shown in Fig. 6(s) and Fig. 6(t) respectively. The difference between the two cases is that the intersections $\boldsymbol{c}^{0,1}$ and $\boldsymbol{c}^{1,0}$ are on opposite sides of the grid line $k_{j+3}$, as the sign of $\{\alpha\}+\{\beta\}-p^{-1}$ changes, cf. (4.25). For $\{\alpha\}+\{\beta\}>p^{-1}$, line $k_{j+3}$ lies below the diagonal, as can be seen from (4.11) and (4.14), so that its configuration $\mathrm{C}(19)$ has 7 odd sites and 5 even sites; for $\{\alpha\}+\{\beta\}<p^{-1}, k_{j+3}$ is above the diagonal, and $\mathrm{C}(20)$ has 5 odd sites and 7 even sites.

For $p^{-1}-p\{\alpha\}<\{\beta\}<p^{-2}-p^{-1}\{\alpha\}$, intersections $\boldsymbol{a}^{1,1}$ and $\boldsymbol{c}^{1,0}$ are no longer inside $P\left(k_{j}, k_{j+1}\right)$. As a consequence two of the even sites are now outside, leaving $\mathrm{C}(21)$ shown in Fig. 6(u) with 5 odd sites and 5 even. For $p^{-2}-p^{-1}\{\alpha\}<\{\beta\}<p^{-1}-p\{\alpha\}$, however, intersections $\boldsymbol{b}^{1,1}$ and $\boldsymbol{c}^{0,1}$ are outside $P\left(k_{j}, k_{j+1}\right)$ instead, such that two different even sites are now outside. The resulting configuration $\mathrm{C}(22)$ shown in Fig. 6(v) has the same 5 odd sites as $\mathrm{C}(21)$, but a different set of 5 even sites. Again, $\mathrm{C}(21)$ and $\mathrm{C}(22)$ are related by the aforementioned reflection symmetry.

For $\{\beta\}<p^{-2}-p^{-1}\{\alpha\}$ and $\{\beta\}<p^{-1}-p\{\alpha\}$, only two intersections $\boldsymbol{a}^{0,1}$ and $\boldsymbol{b}^{0,1}$ are still inside $P\left(k_{j}, k_{j+1}\right)$. These are the cases $\mathrm{C}(23)$ and $\mathrm{C}(24)$ shown in Fig. [6(w) and Fig. [6(x). For $\{\beta\}+\{\alpha\}>p^{-3}$, configuration $\mathrm{C}(23)$, shown in Fig. 6(w), has eight sites: 5 odd and 3 even. Finally, for $0<\{\beta\}<p^{-3}-\{\alpha\}$, the grid line $k_{j+3}+1$ is inside $P$, so that $\mathrm{C}(24)$ shown in Fig. 6( $\mathrm{x}$ ) has nine sites, of which the 5 odd sites are identical to those of $\mathrm{C}(20)$ through $\mathrm{C}(23)$.

In summary, the boundaries for the above 24 regions are the 13 lines given 
by

$$
\begin{aligned}
& \{\alpha\}=p^{-1}, \quad\{\beta\}=p^{-1}, \\
& \{\beta\}+\{\alpha\}=p^{-3}, p^{-1}, 1,2 p^{-1}, \text { or } p, \\
& \{\beta\}+p^{-1}\{\alpha\}=p^{-2}, p^{-1}, \text { or } 1, \\
& \{\beta\}+p\{\alpha\}=p^{-1}, 1, \text { or } p,
\end{aligned}
$$

which are also the boundaries of the inequalities in (4.16), (4.18), (4.22), (4.23) and (4.24), together with the two conditions in (4.25). These are exactly all conditions for three grid lines to meet at a corner of $P$, on an edge of $P$, or inside of $P$, respectively, i.e. the only conditions under which some mesh can appear or disappear in $P$ under shifts of grid lines.

In Fig. 7(a), we plot the boundaries lines given by (4.26) in the unit square with $\{\alpha\}$ and $\{\beta\}$ along the horizontal and vertical axes. These lines indeed divide the unit square into 24 regions. Each of these 24 regions corresponds to a different configuration of the parallelogram $P\left(k_{j}, k_{j+1}\right)$. The above analysis shows that the parallelograms can only have $6,7,8,9,10$ or 12 sites inside. The position of $\left\{\hat{\alpha}\left(k_{j+1}\right)\right\}$ and $\left\{\hat{\beta}\left(k_{j}\right)\right\}$ in the unit square shown in Fig. 7 (a) determines which configuration the parallelogram $P\left(k_{j}, k_{j+1}\right)$ is in.

The areas of the 24 regions in Fig. 7 (a) can be easily calculated. We find, after using the formula of the area of a triangle in terms of the coordinates of its three vertices,

$$
\begin{aligned}
& A(1)=A(2)=\frac{5}{2}-\frac{3}{2} p=\frac{1}{2} p^{-4}, \\
& A(3)=A(5)=A(6)=A(8)=A(9)=A(11)=A(12) \\
& =A(14)=A(19)=A(20)=\frac{5}{2} p-4=\frac{1}{2} p^{-5}, \\
& A(4)=A(7)=A(10)=A(13)=A(15)=A(17)=A(18) \\
& =A(21)=A(22)=A(24)=\frac{13}{2}-4 p=\frac{1}{2} p^{-6}, \\
& A(16)=A(23)=9 p-\frac{29}{2}=\frac{1}{2} p^{-3}-p^{-6} \text {. }
\end{aligned}
$$

The areas of all 22 triangular areas differ by powers of the golden ratio $p$.

For later reference, we display in Fig. 7(b) the eight different regions with equivalent odd configurations. Two odd configurations are equivalent, 


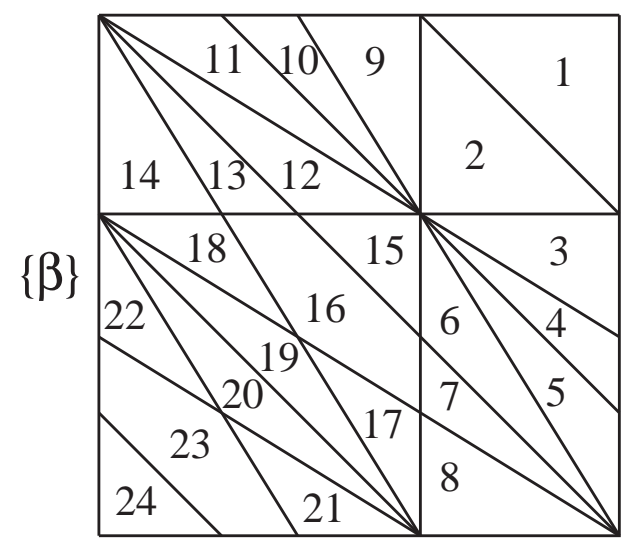

$\{\alpha\}$

(a)

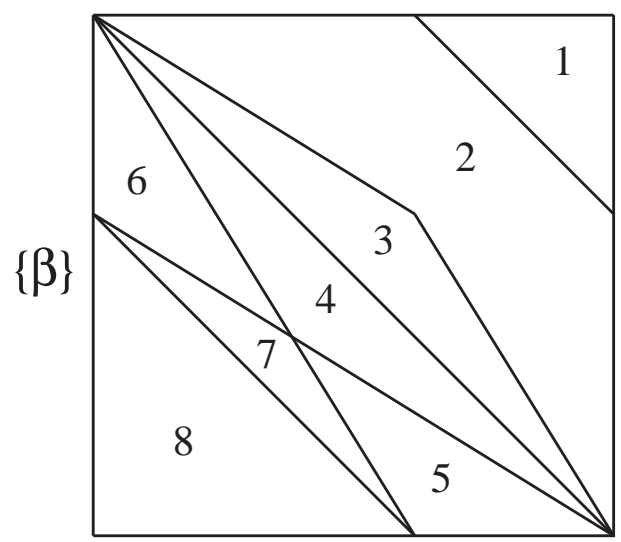

$\{\alpha\}$

(b)

Fig. 7. (a) The 24 regions for the 24 different configurations are shown within the unit square with $\{\alpha\}$ and $\{\beta\}$ along the horizontal and vertical axes. The integer $m$ denotes the $m$ th region for the $m$ th configuration. (b) The 8 regions for the 8 different odd configurations are shown.

if they have an equal number of odd sites with the same sets of integer vectors for these odd sites. This identification can most easily be made using the information in Tables III(a) and III(b) below.

\subsection{Probability}

From the definition (4.4), we find that the fractional parts $\left\{\hat{\alpha}\left(k_{j+1}\right)\right\}$ and $\left\{\hat{\beta}\left(k_{j}\right)\right\}$ are related to the golden ratio $p$ which is irrational. From the wellknown theorem of Kronecker, ${ }^{(62)}$ we conclude that $\left\{\hat{\alpha}\left(k_{j+1}\right)\right\}$ and $\left\{\hat{\beta}\left(k_{j}\right)\right\}$ are everywhere dense and uniformly distributed in the interval $(0,1)$, as the integers $k_{j}$ and $k_{j+1}$ vary from $-\infty$ to $\infty$. As a consequence, every point in the unit square in Fig. 7 is equally probable. Therefore, the frequency or probability for a parallelogram to be in one of the twenty-four configurations, say $m$, is given by the area $A(m)$ of the $m$ th region.

Although the pentagrids are different for different choices of the shifts $\gamma_{j}$, and the values of $\left\{\hat{\alpha}\left(k_{j+1}\right)\right\}$ and $\left\{\hat{\beta}\left(k_{j}\right)\right\}$ are also different for different $\gamma_{j}$ 's, this does not change the probability distributions of $\left\{\hat{\alpha}\left(k_{j+1}\right)\right\}$ and $\left\{\hat{\beta}\left(k_{j}\right)\right\}$ in the thermodynamic limit of $k_{j}$ and $k_{j+1}$ varying from $-\infty$ to $\infty$. In other words, the area $A(m)$ for the $m$ th configuration is independent of the $\gamma_{j}$ 's, 
and is the same for all regular pentagrids.

\subsection{Difference vectors}

For $\{\alpha\}+\{\beta\}>1$ the grid line $k_{j+3}-1$ is seen from (4.18) to be inside $P\left(k_{j}, k_{j+1}\right)$. Consequently, the mesh below corner $\boldsymbol{\epsilon}=(0,0)$ is odd and $\partial \vec{K}=(0,-1,0)$. For $\{\alpha\}+\{\beta\}<1$, line $k_{j+3}-1$ is outside $P\left(k_{j}, k_{j+1}\right)$, and the mesh at corner $\boldsymbol{\epsilon}=(0,0)$ is even. In this case, its integer vector is identical to the reference vector for $P\left(k_{j}, k_{j+1}\right)$ with $\partial \vec{K}(\mathbf{0})=(0,0,0)$. The

difference vectors $\partial \vec{K}$ for all other meshes in the parallelograms in each of the twenty-four configurations can be easily obtained from Fig. 6, as $\partial K_{j+\mathrm{m}}$ changes its values only when a lines in the $(j+\mathrm{m})$ th grid is crossed.

In Tables III(a) and III(b), we list for each of the 24 configurations all the difference vectors $\partial \vec{K}=\left(\partial K_{j+2}, \partial K_{j+3}, \partial K_{j+4}\right)$, with Table III(a) for $\{\alpha\}+\{\beta\}>1$ and Table III(b) for $\{\alpha\}+\{\beta\}<1$. From these tables one can also immediately read off which regions have equivalent odd (or even) configurations.

\subsection{Average Number $\mathcal{N}$}

We shall now calculate $\mathcal{N}$, which is the average number of spin sites in a parallelogram. The total number of sites can be evaluated by counting all the sites in each parallelograms $P\left(k_{j}, k_{j+1}\right)$, and then adding all of them together for all the $P$ 's. This is equivalent to splitting the summation over all sites into two parts - first summing over all sites in $P$ represented by their integer vectors $\vec{K}(\boldsymbol{\epsilon})$ and then adding all of them for all the parallelograms. Let there be $\mathcal{M}$ lines in each of the five grids, so that there are $\mathcal{M}^{2}$ parallelograms, ignoring boundary effects that cancel in the thermodynamic limit. The average $\mathcal{N}$ then equals the total number of lattice sites divided by $\mathcal{M}^{2}$.

We have already shown that each parallelogram $P\left(k_{j}, k_{j+1}\right)$ is in one of 24 configurations $C(m)$ uniquely determined by the values of $\left\{\hat{\alpha}\left(k_{j+1}\right)\right\}$ and $\left\{\hat{\beta}\left(k_{j}\right)\right\}$. The allowed configurations $C(m)$ have $N(m)=6,7,8,9,10$ or 12 sites inside $P$. The frequency or probability $A(m)$ is defined as the number of parallelograms in the $m$ th configuration divided by the total number of parallelograms. If we let $k_{j}$ and $k_{j+1}$ in $P\left(k_{j}, k_{j+1}\right)$ run over the $\mathcal{M}$ values, each of the parallelograms is counted once.

As $\mathcal{M}$ approaches $\infty$, so that $-\infty<k_{j}, k_{j+1}<\infty$, the values of $\left\{\hat{\alpha}\left(k_{j+1}\right)\right\}$ and $\left\{\hat{\beta}\left(k_{j}\right)\right\}$ are everywhere dense and uniformly distributed ${ }^{(62)}$ between 0 
$\frac{1}{0}$
0
0
$\frac{0}{6}$
00
$\frac{0}{\pi}$
$\frac{10}{2}$
$\frac{1}{4}$

Table III(a). $\{\alpha\}+\{\beta\}>1$

\begin{tabular}{|l|c|c|c|c|c|c|c|c|c|c|c|}
\hline & $\mathrm{C}(1)$ & $\mathrm{C}(2)$ & $\mathrm{C}(3)$ & $\mathrm{C}(4)$ & $\mathrm{C}(5)$ & $\mathrm{C}(6)$ & $\mathrm{C}(9)$ & $\mathrm{C}(10)$ & $\mathrm{C}(11)$ & $\mathrm{C}(12)$ & $\mathrm{C}(15)$ \\
\hline & {$[0,-1,0]$} & {$[0,-1,0]$} & {$[0,-1,0]$} & {$[0,-1,0]$} & {$[0,-1,0]$} & {$[0,-1,0]$} & {$[0,-1,0]$} & {$[0,-1,0]$} & {$[0,-1,0]$} & {$[0,-1,0]$} & {$[0,-1,0]$} \\
$\mathrm{O}$ & {$[1,0,0]$} & {$[1,0,0]$} & {$[1,0,0]$} & {$[1,0,0]$} & {$[1,0,0]$} & {$[1,0,0]$} & {$[1,0,0]$} & {$[1,0,0]$} & {$[1,0,0]$} & {$[1,0,0]$} & {$[1,0,0]$} \\
$\mathrm{d}$ & {$[0,0,1]$} & {$[0,0,1]$} & {$[0,0,1]$} & {$[0,0,1]$} & {$[0,0,1]$} & {$[0,0,1]$} & {$[0,0,1]$} & {$[0,0,1]$} & {$[0,0,1]$} & {$[0,0,1]$} & {$[0,0,1]$} \\
$\mathrm{d}$ & {$[1,-1,1]$} & & & & & & & & & & \\
& & & & & & {$[0,1,0]$} & & & & {$[0,1,0]$} & {$[0,1,0]$} \\
\hline & {$[1,-1,0]$} & {$[1,-1,0]$} & {$[1,-1,0]$} & {$[1,-1,0]$} & {$[1,-1,0]$} & & {$[1,-1,0]$} & & & & \\
$\mathrm{e}$ & & {$[0,0,0]$} & {$[0,0,0]$} & {$[0,0,0]$} & {$[0,0,0]$} & {$[0,0,0]$} & {$[0,0,0]$} & {$[0,0,0]$} & {$[0,0,0]$} & {$[0,0,0]$} & {$[0,0,0]$} \\
$\mathrm{V}$ & {$[0,-1,1]$} & {$[0,-1,1]$} & {$[0,-1,1]$} & & & & {$[0,-1,1]$} & {$[0,-1,1]$} & {$[0,-1,1]$} & & \\
$\mathrm{n}$ & {$[1,0,1]$} & {$[1,0,1]$} & {$[1,0,1]$} & & & & {$[1,0,1]$} & & & & \\
& & & {$[1,0,-1]$} & {$[1,0,-1]$} & {$[1,0,-1]$} & {$[1,0,-1]$} & & & & & {$[1,0,-1]$} \\
& & & & & & & {$[-1,0,1]$} & {$[-1,0,1]$} & {$[-1,0,1]$} & {$[-1,0,1]$} & {$[-1,0,1]$} \\
& & & & & {$[1,1,0]$} & {$[1,1,0]$} & & & & & {$[1,1,0]$} \\
& & & & & & & & & {$[0,1,1]$} & {$[0,1,1]$} & {$[0,1,1]$} \\
\hline
\end{tabular}


Table III(b). $\{\alpha\}+\{\beta\}<1$

\begin{tabular}{|c|c|c|c|c|c|c|c|c|c|c|c|c|c|}
\hline & $\mathrm{C}(7)$ & $\mathrm{C}(8)$ & $\mathrm{C}(13)$ & $\mathrm{C}(14)$ & $\mathrm{C}(16)$ & $\mathrm{C}(17)$ & $\mathrm{C}(18)$ & $\mathrm{C}(19)$ & $\mathrm{C}(20)$ & $\mathrm{C}(21)$ & $\mathrm{C}(22)$ & $\mathrm{C}(23)$ & $\mathrm{C}(24)$ \\
\hline \multirow{7}{*}{$\begin{array}{l}\mathrm{O} \\
\mathrm{d} \\
\mathrm{d}\end{array}$} & {$[1,0,0]$} & {$[1,0,0]$} & {$[1,0,0]$} & {$[1,0,0]$} & {$[1,0,0]$} & {$[1,0,0]$} & {$[1,0,0]$} & {$[1,0,0]$} & & & & & \\
\hline & {$[0,1,0]$} & {$[0,1,0]$} & {$[0,1,0]$} & {$[0,1,0]$} & {$[0,1,0]$} & {$[0,1,0]$} & {$[0,1,0]$} & {$[0,1,0]$} & {$[0,1,0]$} & {$[0,1,0]$} & {$[0,1,0]$} & {$[0,1,0]$} & {$[0,1,0]$} \\
\hline & {$[0,0,1]$} & {$[0,0,1]$} & {$[0,0,1]$} & {$[0,0,1]$} & {$[0,0,1]$} & {$[0,0,1]$} & {$[0,0,1]$} & {$[0,0,1]$} & & & & & \\
\hline & & {$[0,0,-1]$} & & & & {$[0,0,-1]$} & & {$[0,0,-1]$} & {$[0,0,-1]$} & {$[0,0,-1]$} & {$[0,0,-1]$} & {$[0,0,-1]$} & {$[0,0,-1]$} \\
\hline & & {$[1,1,-1]$} & & & & {$[1,1,-1]$} & & {$[1,1,-1]$} & {$[1,1,-1]$} & {$[1,1,-1]$} & {$[1,1,-1]$} & {$[1,1,-1]$} & {$[1,1,-1]$} \\
\hline & & & & {$[-1,0,0]$} & & & {$[-1,0,0]$} & {$[-1,0,0]$} & {$[-1,0,0]$} & {$[-1,0,0]$} & {$[-1,0,0]$} & {$[-1,0,0]$} & {$[-1,0,0]$} \\
\hline & & & & {$[-1,1,1]$} & & & {$[-1,1,1]$} & {$[-1,1,1]$} & {$[-1,1,1]$} & {$[-1,1,1]$} & {$[-1,1,1]$} & {$[-1,1,1]$} & {$[-1,1,1]$} \\
\hline \multirow{8}{*}{$\begin{array}{l}\mathrm{e} \\
\mathrm{v}\end{array}$} & {$[0,0,0]$} & {$[0,0,0]$} & {$[0,0,0]$} & {$[0,0,0]$} & {$[0,0,0]$} & {$[0,0,0]$} & {$[0,0,0]$} & {$[0,0,0]$} & {$[0,0,0]$} & {$[0,0,0]$} & {$[0,0,0]$} & {$[0,0,0]$} & {$[0,0,0]$} \\
\hline & & & {$[0,1,1]$} & {$[0,1,1]$} & {$[0,1,1]$} & {$[0,1,1]$} & {$[0,1,1]$} & {$[0,1,1]$} & {$[0,1,1]$} & & {$[0,1,1]$} & & \\
\hline & {$[1,1,0]$} & {$[1,1,0]$} & & & {$[1,1,0]$} & {$[1,1,0]$} & {$[1,1,0]$} & {$[1,1,0]$} & {$[1,1,0]$} & {$[1,1,0]$} & & & \\
\hline & & & & & & & & & {$[-1,1,0]$} & {$[-1,1,0]$} & {$[-1,1,0]$} & {$[-1,1,0]$} & {$[-1,1,0]$} \\
\hline & & & & & & & & & {$[0,1,-1]$} & {$[0,1,-1]$} & {$[0,1,-1]$} & {$[0,1,-1]$} & {$[0,1,-1]$} \\
\hline & {$[1,0,-1]$} & {$[1,0,-1]$} & & & {$[1,0,-1]$} & {$[1,0,-1]$} & {$[1,0,-1]$} & {$[1,0,-1]$} & {$[1,0,-1]$} & {$[1,0,-1]$} & & & \\
\hline & & & {$[-1,0,1]$} & {$[-1,0,1]$} & {$[-1,0,1]$} & {$[-1,0,1]$} & {$[-1,0,1]$} & {$[-1,0,1]$} & {$[-1,0,1]$} & & {$[-1,0,1]$} & & \\
\hline & & & & & & & & & & & & & {$[0,2,0]$} \\
\hline
\end{tabular}


and 1. The frequency $A(m)$ is the area of the $m$ th region in the unit square in Fig. [7(a). The values of these $A(m)$ 's are listed in (4.27). Denoting the number of sites in the $m$ th configuration by $N(m)$, with values also given in the captions of Fig. 6, then the average number of sites per parallelogram is

$$
\begin{aligned}
\mathcal{N}= & \frac{1}{\mathcal{M}^{2}} \sum_{\vec{K}(z \in \mathbb{C})} 1=\frac{1}{\mathcal{M}^{2}} \sum_{\text {all } P} \sum_{\vec{K}(z \in P)} 1 \\
& \stackrel{\mathcal{M} \rightarrow \infty}{\longrightarrow} \sum_{m=1}^{24} A(m) \sum_{n=1}^{N(m)} 1=\sum_{m=1}^{24} A(m) N(m)=5 p .
\end{aligned}
$$

where we have used the notation $\vec{K}(z \in \mathbb{C})$ to denote the integer vectors of all the meshes in the pentagrid, while $\vec{K}(z \in P)$ denotes only those meshes in parallelogram $P$.

\subsection{Penrose Tiles}

The above method provides an alternative way to draw the Penrose tiles. To illustrate this, we let $j=0$. For some fixed shifts $\gamma_{j}$, we let $-J \leqslant k_{0}, k_{1} \leqslant J$ for some positive integer $J$. For any values $k_{0}$ and $k_{1}$ in this set, $\left\{\hat{\alpha}\left(k_{1}\right)\right\}$, $\left\{\hat{\beta}\left(k_{0}\right)\right\}$ are uniquely determined from (4.4). The elements $k_{\mathrm{m}}$, for $\mathrm{m}=2$, 3,4 , of the reference vector in $P\left(k_{0}, k_{1}\right)$ are given by (4.3) and (4.5). From the values of $\left\{\hat{\alpha}\left(k_{1}\right)\right\}$ and $\left\{\hat{\beta}\left(k_{0}\right)\right\}$, we can determine from Fig. 7(a), what configuration $C(m)$ the parallelogram $P\left(k_{0}, k_{1}\right)$ is in. Then we can use Tables III(a) and III(b) to obtain the difference vectors $\partial \vec{K}$ for the $N(m)$ sites inside $P\left(k_{0}, k_{1}\right)$. The integer vectors for these different sites in $P\left(k_{0}, k_{1}\right)$ are then given by

$$
\vec{K}(\boldsymbol{\epsilon})=\left(k_{0}, k_{1}, k_{2}+\partial K_{2}, k_{3}+\partial K_{3}, k_{4}+\partial K_{4}\right)
$$

We use (2.5) and (4.30) to obtain the positions of the spins in the complex plane for the meshes in $P\left(k_{0}, k_{1}\right)$. Hence, as $k_{0}$ and $k_{1}$ run over the values from $-J$ to $J$ we obtain the positions of the spins in both odd and even sublattices shown in Fig. 5. This figure has been plotted using Maple.

\subsection{Summary}

Consider the parallelograms $P\left(k_{j}, k_{j+1}\right)$ which contains all points $z \in \mathbb{C}$ such that $K_{j}(z)=k_{j}$ and $K_{j+1}(z)=k_{j+1}$, cf. (2.4). The configurations 
of two such parallelograms are considered to be the same, if they contain the same number of spin sites and the corresponding sites have the same difference vectors. The different configurations do not depend on the exact locations of the relevant grid lines or their intersections. However, whenever a grid line or an intersection moves in or out of the parallelogram $P$, the configuration changes. The above analysis shows that there are only 24 allowed configurations, with $6,7,8,9,10$ and 12 sites inside $P$.

The configuration of $P\left(k_{j}, k_{j+1}\right)$ is uniquely determined by the values of $\left\{\hat{\alpha}\left(k_{j+1}\right)\right\}$ and $\left\{\hat{\beta}\left(k_{j}\right)\right\}$ defined in (4.4). By examining the locations of the relevant grid lines and their intersections, we find that the unit square, with $\{\alpha\}$ and $\{\beta\}$ along the horizontal and vertical axes, is divided into 24 regions, corresponding to the 24 possible configurations of the parallelogram $P$. This is shown Fig. 7(a), and the 24 configurations of $P$ are shown in Fig. 6, Using the theorem of Kronecker, ${ }^{(62)}$ we find that the area $A(m)$ of the $m$ th region is actually proportional to the probability for the $m$ th configuration to occur. Even though the pentagrids and configurations of the $P\left(k_{j}, k_{j+1}\right)$ 's are different for different choices of the shifts $\gamma_{j}$, the area $A(m)$ is independent of these shifts and is the same for all regular pentagrids.

In each parallelogram $P\left(k_{j}, k_{j+1}\right)$, a reference integer vector is chosen whose components are given by (4.3) and (4.5). The difference vectors with respect to this reference vector are defined in (4.9) and calculated for all of the sites inside $P$. The number of sites $N(m)$, the area $A(m)$, and the difference vectors $\partial \vec{K}$ for the $N(m)$ spin sites for $m=1 \ldots 24$ are listed in Fig. 6, Eq. (4.27) and Tables III(a) and III(b), respectively.

\section{Susceptibility}

There are three $Z$-invariant Ising models that can be defined on the vertices of the Penrose rhombus tiles using the prescriptions of Section 2, Model 1 has spins on all odd sites only, interacting along the diagonals of the tiles, as is illustrated in Fig. 5. Model 2 is defined similarly with spins only on the even sites. Model 3 has all sites of the Penrose tiling, but the even and odd sites are decoupled, with the odd spins interacting as in model 1 and the even spins as in model 2. We will see that the three models have the identical wavevector-dependent susceptibility $\chi(\mathbf{q})$ per spin site in the thermodynamic limit.

The physical positions of the spins have been expressed in (2.5) as complex 
numbers depending on the integer vectors $\vec{K}(z)$ of the meshes. Let $q$ be a complex number and $q=q_{x}+\mathrm{i} q_{y}$ so that $q^{*}$ denotes its complex conjugate (while $\mathbf{q}=\left(q_{x}, q_{y}\right)$ ), then the $\mathbf{q}$-dependent susceptibility is

$$
\begin{aligned}
& k_{\mathrm{B}} T \chi(\mathbf{q})=\lim _{\mathcal{M} \rightarrow \infty} \frac{1}{\mathcal{N} \mathcal{M}^{2}} \sum_{\vec{K}(z \in \mathbb{C})} \sum_{\vec{K}\left(z^{\prime} \in \mathbb{C}\right)} \cos \operatorname{Re}\left\{q^{*} \sum_{j=0}^{4}\left[K_{j}\left(z^{\prime}\right)-K_{j}(z)\right] \zeta^{j}\right\} \\
& \quad \times\left[\left\langle\sigma_{\vec{K}(z)} \sigma_{\vec{K}\left(z^{\prime}\right)}\right\rangle-\left\langle\sigma_{\vec{K}(z)}\right\rangle\left\langle\sigma_{\vec{K}\left(z^{\prime}\right)}\right\rangle\right]= \begin{cases}2 \hat{\chi}^{\mathrm{o}}(\mathbf{q}), & (\operatorname{model} 1), \\
2 \hat{\chi}^{\mathrm{e}}(\mathbf{q}), & (\operatorname{model} 2), \\
\hat{\chi}^{\mathrm{o}}(\mathbf{q})+\hat{\chi}^{\mathrm{e}}(\mathbf{q}), & (\text { model 3). }\end{cases}
\end{aligned}
$$

Here the double sums denoted by $\vec{K}(z \in \mathbb{C})$ are over all the odd spin sites in model 1 , over all the even sites in model 2 , and over all sites in model 3 . In the last case, since the spins on the odd sublattice do not interact with those on the even sublattice, the $\mathbf{q}$-dependent susceptibility $\chi(\mathbf{q})$ becomes the sum of two parts: $\hat{\chi}^{\mathrm{o}}(\mathbf{q})$ denoting the contribution from the odd sublattice and $\hat{\chi}^{\mathrm{e}}(\mathbf{q})$ from the even sublattice. For model 3 the average number of spin sites per parallelogram was given in (4.29) as $\mathcal{N}=5 p$. For models 1 and 2 this number becomes $\mathcal{N}=5 p / 2$, explaining the extra factors 2 in the last member of (5.1).

We shall first consider $\hat{\chi}^{\mathrm{o}}(\mathbf{q})$ and show later that $\hat{\chi}^{\mathrm{e}}(\mathbf{q})$ is equal to it, implying that the susceptibilities of the three models are indeed equal.

\subsection{Calculation of $\chi^{\circ}(q)$}

We again split the sum over all odd spin sites into two parts as in (4.28), and let $\vec{K}^{\circ}(z \in P)$ run over all odd spins in parallelogram $P$. Consequently,

$$
\hat{\chi}^{\mathrm{o}}(\mathbf{q})=\lim _{\mathcal{M} \rightarrow \infty} \frac{1}{\mathcal{N M}^{2}} \sum_{\text {all } P} \sum_{\vec{K}^{\circ}(z \in P)} \sum_{\text {all } P^{\prime}} \sum_{\vec{K}^{\circ}\left(z^{\prime} \in P^{\prime}\right)} U\left(\vec{K}^{\mathrm{o}}\left(z^{\prime}\right), \vec{K}^{\mathrm{o}}(z)\right),
$$

where

$$
\begin{aligned}
U\left(\vec{K}^{\mathrm{o}}\left(z^{\prime}\right), \vec{K}^{\mathrm{o}}(z)\right) & =\left\langle\sigma_{\vec{K}^{\circ}(z)} \sigma_{\vec{K}^{\circ}\left(z^{\prime}\right)}\right\rangle^{\mathrm{c}} \\
& \times \cos \operatorname{Re}\left\{q^{*} \sum_{n=0}^{4}\left[K_{n}^{\mathrm{o}}\left(z^{\prime}\right)-K_{n}^{\mathrm{o}}(z)\right] \zeta^{n}\right\}
\end{aligned}
$$


with $\left\langle\sigma \sigma^{\prime}\right\rangle^{\mathrm{c}}$ denoting the connected pair correlation function, subtracting the contribution from the spontaneous magnetization.

Now we let $P=P\left(k_{j}, k_{j+1}\right)$ and $P^{\prime}=P\left(k_{j}+\ell, k_{j+1}+\ell^{\prime}\right)$. As $\mathcal{M} \rightarrow \infty$, $\ell$ and $\ell^{\prime}$ are kept fixed, and $k_{j}$ and $k_{j+1}$ vary from $-\infty$ to $\infty$, all the $P^{\prime}$ s and $P^{\prime}$ 's are counted once. It is also evident that the different choices of $j$ correspond to choosing one of five orientations for the parallelograms, and they should give the same q-dependent susceptibility.

In (4.2) we defined $z=z(\boldsymbol{\epsilon})$ for $z \in P$. Similarly, for $z^{\prime} \in P^{\prime}$, we let

$$
z^{\prime}=\frac{\mathrm{i}\left[\zeta^{j}\left(k_{j+1}+\ell^{\prime}-\gamma_{j+1}-\epsilon_{j+1}^{\prime}\right)-\zeta^{j+1}\left(k_{j}+\ell-\gamma_{j}-\epsilon_{j}^{\prime}\right)\right]}{\sin (2 \pi / 5)} \equiv z^{\prime}\left(\boldsymbol{\epsilon}^{\prime}\right),
$$

so that $z^{\prime} \leftrightarrow \boldsymbol{\epsilon}^{\prime}=\left(\epsilon_{j}^{\prime}, \epsilon_{j+1}^{\prime}\right)$ and $0<\epsilon_{j}^{\prime}, \epsilon_{j+1}^{\prime}<1$. The corresponding integer vectors $\vec{K}\left(z^{\prime}\right)=\vec{K}\left(z^{\prime}\left(\boldsymbol{\epsilon}^{\prime}\right)\right) \equiv \vec{K}^{\prime}\left(\boldsymbol{\epsilon}^{\prime}\right)$ also have two fixed components $K_{j}^{\prime}\left(\boldsymbol{\epsilon}^{\prime}\right)=$ $k_{j}+\ell \equiv k_{j}^{\prime}$ and $K_{j+1}^{\prime}\left(\boldsymbol{\epsilon}^{\prime}\right)=k_{j+1}+\ell^{\prime} \equiv k_{j+1}^{\prime}$. Now, following (4.4), we let

$$
\begin{aligned}
& \alpha^{\prime} \equiv \hat{\alpha}\left(k_{j+1}^{\prime}\right)=p^{-1}\left(k_{j+1}+\ell^{\prime}-\gamma_{j+1}\right)+\gamma_{j}+\gamma_{j+2}=\alpha+p^{-1} \ell^{\prime}, \\
& \beta^{\prime} \equiv \hat{\beta}\left(k_{j}^{\prime}\right)=p^{-1}\left(k_{j}+\ell-\gamma_{j}\right)+\gamma_{j+4}+\gamma_{j+1}=\beta+p^{-1} \ell .
\end{aligned}
$$

According to (4.3) and (4.5) the reference integer vector $\vec{k}^{\prime}$ for $P^{\prime}$ is chosen to have components,

$$
\begin{aligned}
& k_{j+2}^{\prime}=\left\lceil\alpha^{\prime}\right\rceil-k_{j}-\ell=k_{j+2}+\delta_{j+2}+\left\lfloor p^{-1} \ell^{\prime}\right\rfloor-\ell, \\
& k_{j+3}^{\prime}=-\left\lfloor\alpha^{\prime}\right\rfloor-\left\lfloor\beta^{\prime}\right\rfloor=k_{j+3}-\delta_{j+2}-\left\lfloor p^{-1} \ell^{\prime}\right\rfloor-\delta_{j+4}-\left\lfloor p^{-1} \ell\right\rfloor, \\
& k_{j+4}^{\prime}=\left\lceil\beta^{\prime}\right\rceil-k_{j+1}-\ell^{\prime}=k_{j+4}+\delta_{j+4}+\left\lfloor p^{-1} \ell\right\rfloor-\ell^{\prime},
\end{aligned}
$$

in which 8

$$
\delta_{j+2}=\left\lfloor\{\alpha\}+\left\{p^{-1} \ell^{\prime}\right\}\right\rfloor, \quad \delta_{j+4}=\left\lfloor\{\beta\}+\left\{p^{-1} \ell\right\}\right\rfloor .
$$

Substituting (5.4) into (2.4) we obtain for $\mathrm{m}=2,3,4$

$$
K_{j+\mathrm{m}}^{\prime}\left(\boldsymbol{\epsilon}^{\prime}\right)=k_{j+\mathrm{m}}^{\prime}+\partial K_{j+\mathrm{m}}^{\prime}\left(\boldsymbol{\epsilon}^{\prime}\right), \quad \partial K_{j+\mathrm{m}}^{\prime}\left(\boldsymbol{\epsilon}^{\prime}\right)=\left\lfloor\lambda_{j+\mathrm{m}}^{\prime}\left(\boldsymbol{\epsilon}^{\prime}\right)\right\rfloor,
$$

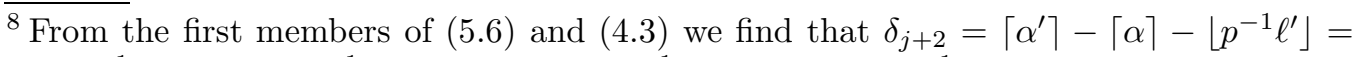
$\left\lceil\alpha+p^{-1} \ell^{\prime}\right\rceil-\lceil\alpha\rceil-\left\lfloor p^{-1} \ell^{\prime}\right\rfloor=\left\lceil\{\alpha\}-1+\left\{p^{-1} \ell^{\prime}\right\}\right\rceil=\left\lfloor\{\alpha\}+\left\{p^{-1} \ell^{\prime}\right\}\right\rfloor$. For the last two steps here and the remaining steps in the derivation of (5.6) and (5.7) we must make explicit use of the fact that $\alpha, \alpha^{\prime}, \beta$ and $\beta^{\prime}$ are not integer for a regular pentagrid.
} 
where

$$
\lambda_{j+2}^{\prime}\left(\boldsymbol{\epsilon}^{\prime}\right)=\left\{\alpha^{\prime}\right\}+\epsilon_{j}^{\prime}-p^{-1} \epsilon_{j+1}^{\prime}, \quad \lambda_{j+4}^{\prime}\left(\boldsymbol{\epsilon}^{\prime}\right)=\left\{\beta^{\prime}\right\}+\epsilon_{j+1}^{\prime}-p^{-1} \epsilon_{j}^{\prime} .
$$

and

$$
\lambda_{j+3}^{\prime}\left(\boldsymbol{\epsilon}^{\prime}\right)=p^{-1}\left(\epsilon_{j}^{\prime}+\epsilon_{j+1}^{\prime}\right)-\left\{\alpha^{\prime}\right\}-\left\{\beta^{\prime}\right\}+1 .
$$

Comparing (5.8) to (5.10) with (4.9) to (4.11), we find that the dependence of $\lambda_{j+\mathrm{m}}^{\prime}\left(\boldsymbol{\epsilon}^{\prime}\right)$ on $\left\{\alpha^{\prime}\right\}$ and $\left\{\beta^{\prime}\right\}$ is the same as the dependence of $\lambda_{j+\mathrm{m}}(\boldsymbol{\epsilon})$ on $\{\alpha\}$ and $\{\beta\}$. Consequently, the configurations of $P^{\prime}$ depend on $\left\{\alpha^{\prime}\right\}$ and $\left\{\beta^{\prime}\right\}$ in the same way as the configurations of $P$ on $\{\alpha\}$ and $\{\beta\}$. Therefore, the position of $\left\{\alpha^{\prime}\right\}$ and $\left\{\beta^{\prime}\right\}$ in the unit square in Fig. 7(a) with $\left\{\alpha^{\prime}\right\}$ and $\left\{\beta^{\prime}\right\}$ along the horizontal and vertical axes uniquely determines the configuration of $P^{\prime}$. The difference vectors $\partial \vec{K}^{\prime}\left(\boldsymbol{\epsilon}^{\prime}\right)$ for the sites in $P^{\prime}$, which is in one of the 24 configurations, are again given in Tables III(a) and III(b).

The above results are valid for all spin configurations in $P$ and $P^{\prime}$, but we shall consider only the odd spins at first. By examining Tables III(a) and III(b), we can find that the odd spin configurations of the parallelograms are simpler, because several connected regions - see $\mathrm{C}(2)$ to $\mathrm{C}(5)$, or $\mathrm{C}(9)$ to $\mathrm{C}(11)$ in Fig. 7(a) as examples - have the same odd spin configurations. In fact, there are only eight distinct odd spin configurations. In Fig. 7 (b), the regions for these 8 odd spin configurations are shown in the unit square whose axes are the $\{\alpha\}$ and $\{\beta\}$ directions.

Listed in Table IV are the number of odd sites $\hat{N}(m)$ in $P$, the region of validity $R(m)$, and the area $\hat{A}(m)$ of the $m$ th odd configuration for all $m=1, \cdots, 8$. In the $m$ th odd configuration, the difference vectors of the $\hat{N}(m)$ spins are denoted by $\partial \vec{K}^{[m, n]}$ for $n=1, \cdots, \hat{N}(m)$. They are equal to the difference vectors $\partial \vec{K}^{\circ}$ of the odd sites in some configuration $\mathrm{C}(l)$, listed in Tables III(a) and III(b) for $l=1, \cdots, 24$. In the last column of Table IV it is indicated which $\mathrm{C}(l)$ 's correspond to a given $m$.

Let the distances $\ell$ and $\ell^{\prime}$ between the two parallelograms $P$ and $P^{\prime}$ be fixed, but $k_{j}$ and $k_{j+1}$ vary from $-\infty$ to $\infty$. Then $\left\{\alpha^{\prime}\right\}$ and $\left\{\beta^{\prime}\right\}$ given by (5.5) are also everywhere dense and uniformly distributed in the interval $(0,1)$. The area $\hat{A}(m)$ is again the probability or frequency of the $m$ th configuration. 
Table IV. The eight regions for the odd sublattice

\begin{tabular}{|c|c|c|c|c|}
\hline$m$ & $\hat{N}(m)$ & $\hat{A}(m)$ & $R(m)$ & $\begin{array}{l}\partial \vec{K}^{[m, n]}=\partial \vec{K}^{\mathrm{o}} \\
1 \leqslant n \leqslant \hat{N}(m)\end{array}$ \\
\hline 1 & 4 & $\frac{1}{2} p^{-4}$ & $p^{-1}<\{\alpha\}<1 \& p-\{\alpha\}<\{\beta\}<1$ & $\partial \vec{K}^{\mathrm{o}}$ in $\mathrm{C}(1)$ \\
\hline 2 & 3 & $\frac{1}{2} p^{-1}$ & $\begin{array}{l}0<\{\alpha\} \leqslant p^{-1} \& 1-p^{-1}\{\alpha\}<\{\beta\}<1 \\
p^{-1} \leqslant\{\alpha\}<1 \& p(1-\{\alpha\})<\{\beta\}<p-\{\alpha\}\end{array}$ & $\begin{array}{l}\partial \vec{K}^{\mathrm{o}} \text { in } \mathrm{C}(2) \text { or } \\
\mathrm{C}(l), l=3,4,5,9,10,11\end{array}$ \\
\hline 3 & 4 & $\frac{1}{2} p^{-3}$ & $\begin{array}{l}0<\{\alpha\} \leqslant p^{-1} \& 1-\{\alpha\}<\{\beta\}<1-p^{-1}\{\alpha\} \\
p^{-1} \leqslant\{\alpha\}<1 \& 1-\{\alpha\}<\{\beta\}<p(1-\{\alpha\})\end{array}$ & $\begin{array}{l}\partial \vec{K}^{\mathrm{o}} \text { in } \mathrm{C}(6) \\
\mathrm{C}(12) \text { or } \mathrm{C}(15)\end{array}$ \\
\hline 4 & 3 & $\frac{1}{2} p^{-3}$ & $\begin{array}{l}0<\{\alpha\} \leqslant p^{-2} \& 1-p\{\alpha\}<\{\beta\}<1-\{\alpha\} \\
p^{-2} \leqslant\{\alpha\}<1 \& p^{-1}(1-\{\alpha\})<\{\beta\}<1-\{\alpha\}\end{array}$ & $\begin{array}{l}\partial \vec{K}^{\mathrm{o}} \text { in } \mathrm{C}(7) \\
\mathrm{C}(13) \text { or } \mathrm{C}(16)\end{array}$ \\
\hline 5 & 5 & $\frac{1}{2} p^{-4}$ & $0<\{\beta\}<p^{-2} \& p^{-1}(1-\{\beta\})<\{\alpha\}<1-p\{\beta\}$ & $\partial \vec{K}^{\mathrm{o}}$ in $\mathrm{C}(8)$ or $\mathrm{C}(17)$ \\
\hline 6 & 5 & $\frac{1}{2} p^{-4}$ & $0<\{\alpha\}<p^{-2} \& p^{-1}(1-\{\alpha\})<\{\beta\}<1-p\{\alpha\}$ & $\partial \vec{K}^{\mathrm{o}}$ in $\mathrm{C}(14)$ or $\mathrm{C}(18)$ \\
\hline 7 & 7 & $\frac{1}{2} p^{-5}$ & $\begin{array}{l}0<\{\alpha\} \leqslant p^{-2} \& p^{-1}-\{\alpha\}<\{\beta\}<p^{-1}(1-\{\alpha\}) \\
p^{-2} \leqslant\{\alpha\}<p^{-1} \& p^{-1}-\{\alpha\}<\{\beta\}<1-p\{\alpha\}\end{array}$ & $\partial \vec{K}^{\mathrm{o}}$ in $\mathrm{C}(19)$ \\
\hline 8 & 5 & $\frac{1}{2} p^{-2}$ & $0<\{\alpha\}<p^{-1} \& 0<\{\beta\}<p^{-1}-\{\alpha\}$ & $\partial \vec{K}^{\mathrm{o}}$ in $\mathrm{C}(20)$ to $\mathrm{C}(24)$ \\
\hline
\end{tabular}


From (5.5) we find

$$
\begin{aligned}
& \left\{\alpha^{\prime}\right\}=\left\{\begin{array}{lll}
\{\alpha\}+a & \text { for } & \{\alpha\}+a<1 \\
\{\alpha\}+a-1 & \text { for } & \{\alpha\}+a \geqslant 1
\end{array}\right\}, \quad a=\left\{p^{-1} \ell^{\prime}\right\}, \\
& \left\{\beta^{\prime}\right\}=\left\{\begin{array}{lll}
\{\beta\}+b & \text { for } & \{\beta\}+b<1 \\
\{\beta\}+b-1 & \text { for } & \{\beta\}+b \geqslant 1
\end{array}\right\}, \quad b=\left\{p^{-1} \ell\right\} .
\end{aligned}
$$

The plot of the eight regions for the odd configurations of $P=P\left(k_{j}, k_{j+1}\right)$

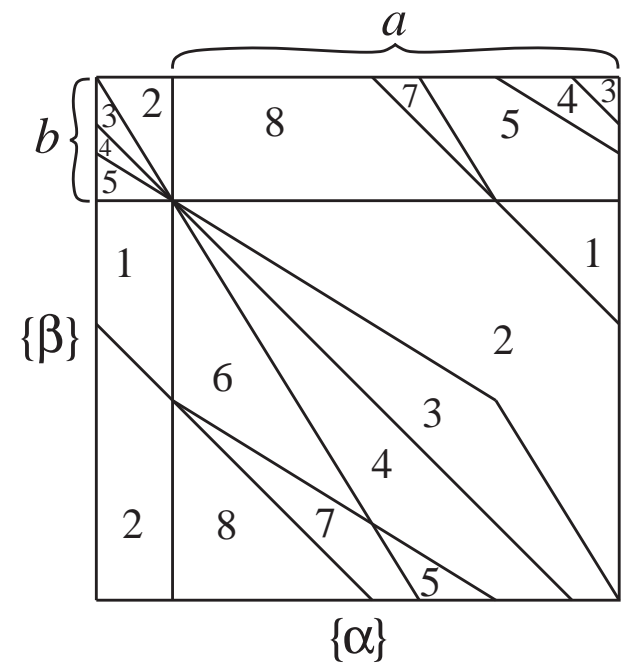

(a)

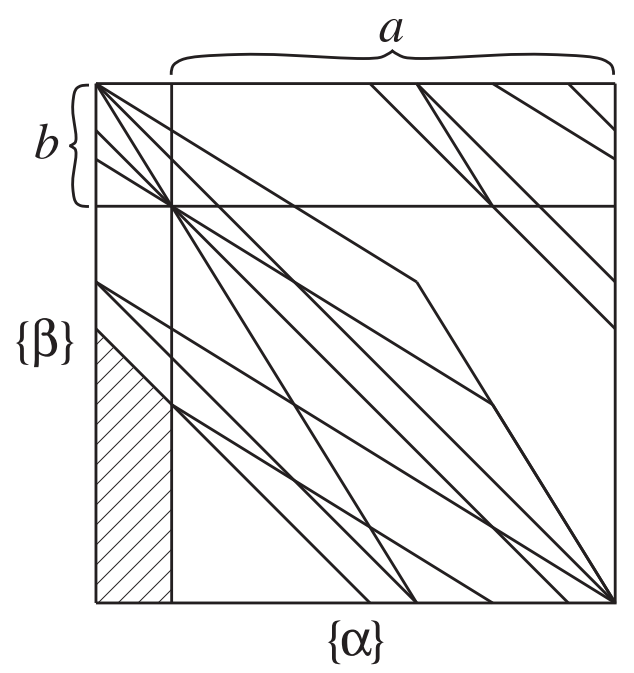

(b)

Fig. 8. (a) The eight odd spin configurations in $P\left(k_{j}+2, k_{j+1}+3\right)$ plotted with $\{\alpha\}$ and $\{\beta\}$ as the horizontal and vertical axes. (b) Overlapping the plot in part (a) with Fig. 7 (b) we can determine the joint probability $A_{m, m^{\prime}}(2,3)$ for $P\left(k_{j}, k_{j+1}\right)$ to be in the $m$ th configuration and $P\left(k_{j}+2, k_{j+1}+3\right)$ in $m^{\prime}$ th configuration, with $m, m^{\prime}=1, \cdots, 8$, geometrically. The area of the shaded region represents the probability for $m=8$ and $m^{\prime}=2$.

has been given in Fig. (7)(b). The plot for $P^{\prime}=P\left(k_{j}+\ell, k_{j+1}+\ell^{\prime}\right)$ is the same, only now with $\left\{\alpha^{\prime}\right\}$ and $\left\{\beta^{\prime}\right\}$ along the axes. However, if the eight regions are replotted with $\{\alpha\}$ and $\{\beta\}$ along the horizontal and vertical axes, then we obtain unit squares as shown in Fig. 8(a) [for the special case of $\ell=2$ and $\ell^{\prime}=3$ ]. Because of the relation (5.11), we find that Fig. 8 (a) can be obtained from Fig. 7 (b) by cutting a horizontal slice with width $b$ from the bottom 
of Fig. 7(b), and pasting it on the top; then cutting a vertical slice of width $a$ from the left and pasting it to the right. After the cutting and pasting, the connected region $R(m)$ for $P^{\prime}$ in Fig. 7(b) becomes $R^{\prime}(m)$ in Fig. 8(a), which-for $\ell \neq 0$ or $\ell^{\prime} \neq 0$-may consist of disjointed pieces pasted in up to four different sections of the unit square. The probability for $P^{\prime}$ to be in the $m$ th configuration is still the area $\hat{A}(m)=\operatorname{area}(R(m))=\operatorname{area}\left(R^{\prime}(m)\right)$, which in the latter case, could be a sum of areas of disjointed pieces.

For fixed $\ell$ and $\ell^{\prime}$ [chosen to be $\ell=2$ and $\ell^{\prime}=3$ in Fig. 8 (a)], the position of $\alpha=\hat{\alpha}\left(k_{j+1}\right)$ and $\beta=\hat{\beta}\left(k_{j}\right)$ in the unit square shown in Fig. [7(b) uniquely determines the configuration of $P\left(k_{j}, k_{j+1}\right)$, while the position of $\alpha^{\prime}=\hat{\alpha}\left(k_{j+1}+\ell^{\prime}\right)$ and $\beta^{\prime}=\hat{\beta}\left(k_{j}+\ell\right)$ in Fig. 8(a) determines the configuration of $P\left(k_{j}+\ell, k_{j+1}+\ell^{\prime}\right)$. As $k_{j}$ and $k_{j+1}$ run over all the values from $-\infty$ to $\infty$, we find from Kronecker's theorem ${ }^{(62)}$ that every point in either Fig. 7(b) or Fig. 8 (a) is equally probable. However, the positions of $(\alpha, \beta)$ and $\left(\alpha^{\prime}, \beta^{\prime}\right)$ are completely correlated by the shift $(a, b)$ in (5.11), which is fixed as long as $\ell$ and $\ell^{\prime}$ are unchanged. Thus, the joint probability for $P\left(k_{j}, k_{j+1}\right)$ to be in the $m$ th configuration and $P\left(k_{j}+\ell, k_{j+1}+\ell^{\prime}\right)$ to be in the $m^{\prime}$ th configuration is the area of the intersection of the two regions $R(m)$ and $R^{\prime}\left(m^{\prime}\right)$, and is denoted by

$$
A_{m, m^{\prime}}\left(\ell, \ell^{\prime}\right)=\operatorname{area}\left(R(m) \cap R^{\prime}\left(m^{\prime}\right)\right) .
$$

By superimposing Fig. 7(b) on top of Fig. 8(a), we obtain Fig. 8(b). This figure gives the intersections of all the regions of Fig. 7 (b) with all the regions of Fig. 8(a), and the joint probabilities can be read off as the areas of these intersections.

For different values of $\ell$ and $\ell^{\prime}$, we get different values of the width $a$ given in (5.11) of the vertical slice in Fig. 8 (a) and also of the width $b$ of the horizontal slice. After cutting and pasting the difference slices, the resulting figures are very different, so are the superimposed figures. Thus, the area of intersection $A_{m, m^{\prime}}\left(\ell, \ell^{\prime}\right)$ depends on the choice of $\ell$ and $\ell^{\prime}$. However, just as $\hat{A}(m)$ and $\hat{A}\left(m^{\prime}\right)$ do not depend on the shifts $\gamma_{j}, A_{m, m^{\prime}}\left(\ell, \ell^{\prime}\right)$ also does not depend on these shifts. Moreover, it is easily seen that this joint probability $A_{m, m^{\prime}}\left(\ell, \ell^{\prime}\right)$ is not only the same for all the different regular pentagrids, but also the same for the different orientations of the parallelograms (i.e. different choices of $j=0, \cdots, 4)$.

We let $\vec{K}^{[m, n]}$ denote the $n$th integer vector in the $m$ th odd configuration for odd spins inside $P$, where $n=1, \cdots, N(m)$ and $m=1, \cdots, 8$. Similarly $\vec{K}^{\left[m^{\prime}, n^{\prime}\right]}$ denotes the $n^{\prime}$ th integer vector of the $m^{\prime}$ th odd configuration of odd 
spins inside $P^{\prime}$, with $n^{\prime}=1, \cdots, N\left(m^{\prime}\right)$ and $m=1, \cdots, 8$. From Tables III(a), III(b) and IV, the possible difference vectors $\partial \vec{K}^{[m, n]}$ and $\partial \vec{K}^{\left[m^{\prime}, n^{\prime}\right]}$ for two spins in $P$ and $P^{\prime}$ may be found. Adding, as in (4.9) and (5.8), these to their corresponding reference integer vectors, with three of their components given in (4.3), (4.5) and (5.6), we obtain the two integer vectors $\vec{K}^{\mathrm{o}}(z)=\vec{K}^{[m, n]}$ for the spin in $P$ and $\vec{K}^{\circ}\left(z^{\prime}\right)=\vec{K}^{\left[m^{\prime}, n^{\prime}\right]}$ for the spin in $P^{\prime}$. In (5.1) and (5.3) we only need their difference

$$
\vec{K}^{\mathrm{o}}\left(z^{\prime}\right)-\vec{K}^{\mathrm{o}}(z)=\vec{K}^{\left[m^{\prime}, n^{\prime}\right]}-\vec{K}^{[m, n]} \equiv\left(\ell_{0}, \ell_{1}, \cdots, \ell_{4}\right) .
$$

We find from (4.9), (5.6) and (5.8) that

$$
\begin{aligned}
& \ell_{j}=\ell, \quad \ell_{j+1}=\ell^{\prime} \\
& \ell_{j+2}=\delta_{j+2}+\left\lfloor p^{-1} \ell^{\prime}\right\rfloor-\ell+\partial K_{j+2}^{\left[m^{\prime}, n^{\prime}\right]}-\partial K_{j+2}^{[m, n]} \equiv \ell^{\prime \prime} \\
& \ell_{j+3}=-\delta_{j+2}-\delta_{j+4}-\left\lfloor p^{-1} \ell^{\prime}\right\rfloor-\left\lfloor p^{-1} \ell\right\rfloor+\partial K_{j+3}^{\left[m^{\prime}, n^{\prime}\right]}-\partial K_{j+3}^{[m, n]} \equiv \ell^{\prime \prime \prime} \\
& \ell_{j+4}=\delta_{j+4}+\left\lfloor p^{-1} \ell\right\rfloor-\ell^{\prime}+\partial K_{j+4}^{\left[m^{\prime}, n^{\prime}\right]}-\partial K_{j+4}^{[m, n]} \equiv \ell^{\prime \prime \prime \prime}
\end{aligned}
$$

It is easy to see from (5.7) and (5.11) that

$$
\begin{aligned}
& \delta_{j+2}=\left\{\begin{array}{lll}
0 & \text { if } & 0 \leqslant\{\alpha\}<1-a, \\
1 & \text { if } & 1-a \leqslant\{\alpha\}<1,
\end{array}\right. \\
& \delta_{j+4}=\left\{\begin{array}{lll}
0 & \text { if } & 0 \leqslant\{\beta\}<1-b, \\
1 & \text { if } & 1-b \leqslant\{\beta\}<1,
\end{array}\right.
\end{aligned}
$$

which shows that in the four sectors of the unit square shown in Fig. 8(b), the $\delta_{i}$ pairs are different. As $a$ and $b$ are functions of $\ell$ and $\ell^{\prime}$ only, and the entries in Tables III(a), III(b) and IV are also independent of $j$, the results in (5.14) are easily seen to be functions of $\ell$ and $\ell^{\prime}$, and of $\{\alpha\}$ and $\{\beta\}$, but they are independent of $j$. Therefore, we use the primed variables $\ell, \cdots, \ell^{\prime \prime \prime \prime}$ to denote these $j$-independent values of $\ell_{j}, \cdots, \ell_{j+4}$, i.e.

$$
\tilde{\ell} \equiv\left[\ell, \ell^{\prime}, \cdots, \ell^{\prime \prime \prime \prime}\right]=\left[\ell_{j}, \ell_{j+1}, \cdots, \ell_{j+4}\right]
$$

For different choices of $j,\left[\ell_{0}, \ell_{1}, \ell_{2}, \ell_{3}, \ell_{4}\right]$ is just a cyclic permutation of $\tilde{\ell}$.

If we let the distance vector $\left(\ell, \ell^{\prime}\right)$ between the two parallelograms $P=$ $P\left(k_{j}, k_{j+1}\right)$ and $P^{\prime}=P\left(k_{j}+\ell, k_{j+1}+\ell^{\prime}\right)$ in (5.2) be fixed, while letting both 
$k_{j}$ and $k_{j+1}$ vary from $-\infty$ to $\infty$ (which is equivalent to $\mathcal{M} \rightarrow \infty$ ), then the parallelograms $P$ and $P^{\prime}$ each are in one of eight different odd configurations. Since the joint probability for $P$ being in the $m$ th configuration and $P^{\prime}$ in the $m^{\prime}$ th configuration is $A_{m, m^{\prime}}\left(\ell, \ell^{\prime}\right)$ given in (5.12), the double sum in (5.2) can be rewritten as

$$
\begin{array}{r}
\hat{\chi}^{\mathrm{o}}(\mathbf{q})=\lim _{\mathcal{M} \rightarrow \infty} \frac{1}{\mathcal{N} \mathcal{M}^{2}} \sum_{\ell, \ell^{\prime}} \sum_{k_{j}, k_{j+1}} \sum_{\vec{K}^{\circ}(\boldsymbol{\epsilon})} \sum_{\vec{K}^{\circ \prime}\left(\boldsymbol{\epsilon}^{\prime}\right)} U\left(\vec{K}^{\mathrm{o}}(\boldsymbol{\epsilon}), \vec{K}^{\mathrm{o} \prime}\left(\boldsymbol{\epsilon}^{\prime}\right)\right) \\
=\frac{1}{\mathcal{N}} \sum_{\ell, \ell^{\prime}} \sum_{m=1}^{8} \sum_{m^{\prime}=1}^{8} A_{m, m^{\prime}}\left(\ell, \ell^{\prime}\right) \sum_{n=1}^{N(m)} \sum_{n^{\prime}=1}^{N\left(m^{\prime}\right)} U\left(\vec{K}^{[m, n]}, \vec{K}^{\left[m^{\prime}, n^{\prime}\right]}\right) .
\end{array}
$$

with $\mathcal{N}=5 p$, cf. (4.29). Using (3.1) and (5.13), we find (5.3) becomes

$$
U\left(\vec{K}^{[m, n]}, \vec{K}^{\left[m^{\prime}, n^{\prime}\right]}\right)=\cos \left[\operatorname{Re}\left(q^{*} \sum_{k=0}^{4} \ell_{k} \zeta^{k}\right)\right]\left\langle\sigma \sigma^{\prime}\right\rangle_{\left[\ell_{0}, \ell_{1}, \cdots, \ell_{4}\right]}^{\mathrm{c}},
$$

which is different for different $j$ in view of (5.16). Since the correlation functions have the cyclic property shown in (3.3), and $\hat{\chi}^{\mathrm{o}}(\mathbf{q})$ can be evaluated by choosing parallelograms $P$ and $P^{\prime}$ in (5.2) oriented in any one of the five directions (any choice of $j$ ), we can rewrite $\hat{\chi}^{\mathrm{o}}\left(q_{x}, q_{y}\right)$ in a more symmetric way by expressing it as the sum over the five different orientations $j$, and then dividing the result by 5 . This means,

$$
\hat{\chi}^{\mathrm{o}}(\mathbf{q})=\sum_{\ell=-\infty}^{\infty} \sum_{\ell^{\prime}=-\infty}^{\infty} \hat{\chi}^{\mathrm{o}}(\mathbf{q})_{\ell, \ell^{\prime}}
$$

where (5.17) to (5.16) and $(\underline{3.3)}$ are used to find

$$
\begin{aligned}
& \hat{\chi}^{\mathrm{O}}(\mathbf{q})_{\ell, \ell^{\prime}}=\frac{1}{\mathcal{N}} \sum_{m=1}^{8} \sum_{m^{\prime}=1}^{8} A_{m, m^{\prime}}\left(\ell, \ell^{\prime}\right) \sum_{n=1}^{N(m)} \sum_{n^{\prime}=1}^{N\left(m^{\prime}\right)} c(\mathbf{q}, \tilde{\boldsymbol{\ell}})\left\langle\sigma \sigma^{\prime}\right\rangle_{\left[\ell, \ell^{\prime}, \ell^{\prime \prime}, \ell^{\prime \prime \prime}, \ell^{\prime \prime \prime \prime}\right]} \\
& c(\mathbf{q}, \tilde{\ell})=\frac{1}{5} \sum_{j=1}^{5} \cos \operatorname{Re}\left[q^{*} \zeta^{j}\left(\ell+\ell^{\prime} \zeta+\ell^{\prime \prime} \zeta^{2}+\ell^{\prime \prime \prime} \zeta^{3}+\ell^{\prime \prime \prime \prime} \zeta^{4}\right)\right]
\end{aligned}
$$

It satisfies the following identities,

$$
\hat{\chi}^{\mathrm{O}}(\mathbf{q})_{-\ell,-\ell^{\prime}}=\hat{\chi}^{\mathrm{O}}(\mathbf{q})_{\ell, \ell^{\prime}}, \quad \hat{\chi}^{\mathrm{O}}\left(\mathbf{q}^{*}\right)_{\ell^{\prime}, \ell}=\hat{\chi}^{\mathrm{O}}(\mathbf{q})_{\ell, \ell^{\prime}}
$$


in which $\mathbf{q}^{*} \leftrightarrow\left(q_{x},-q_{y}\right)$. The former identity in (5.21) is easily seen as a consequence of the reflection symmetry in the correlation function $\left\langle\sigma \sigma^{\prime}\right\rangle=$ $\left\langle\sigma^{\prime} \sigma\right\rangle$; the latter one is due to five-fold rotation and reflection symmetry 9

In the actual calculation, because $\delta_{2}$ and $\delta_{4}$ generally differ in four sectors of the unit square, the contributions to the susceptibility from these different sectors are evaluated separately.

\subsection{Results}

To evaluate the wavevector-dependent susceptibility (5.19), (5.20), we can compute the $A_{m, m^{\prime}}\left(\ell, \ell^{\prime}\right)$ as the overlap area (5.12) and the $\ell^{\prime}$ 's from (5.14). In Table II and Eq. (3.2) in Section 3 we have expressed the pair-correlation function $\left\langle\sigma \sigma^{\prime}\right\rangle_{\left[\ell_{0}, \cdots, \ell_{4}\right]}$ in terms of Baxter's universal functions $g$, ${ }^{(14)}$ which can be evaluated using methods in our earlier work. ${ }^{(53][54] 60)}$

Near the critical point $k=1$ the leading asymptotic behavior of the paircorrelation function is the same Painlevé III or V scaling function $\frac{(53,60)}{6 s}$ in the uniform rectangular lattice. ${ }^{(45)}$ Therefore, the scaling behavior of the central peak of $\chi(\mathbf{q})$ in our Penrose Ising model is also known $\underline{606}$ [6 6 the same as for the regular Ising model.

More interesting is the incommensurate behavior of $\chi(\mathbf{q})$ as a function of wavevector $\mathbf{q}$ and how it changes with temperature or $k$. At the critical point we expect $\chi(\mathbf{q})$ to be a function that has everywhere dense $7 / 4$-th power divergencies, but is locally integrable. It is nontrivial to show this directly, but this conclusion seems to impose itself as one approaches the critical point from either side.

Since the correlation functions decay exponentially away from the critical point, we find that the $\hat{\chi}^{\mathrm{o}}(\mathbf{q})_{\ell, \ell^{\prime}}$ are rapidly decreasing functions of $\ell$ and $\ell^{\prime}$. Putting terms of about the same order of magnitude together, we find

$$
\hat{\chi}^{\mathrm{o}}(\mathbf{q})=\hat{\chi}^{\mathrm{o}}(\mathbf{q})_{0,0}+\sum_{\ell=1}^{\infty} \mathcal{S}_{\ell}, \quad \mathcal{S}_{\ell}=2 \sum_{n=-\ell+1}^{\ell}\left[\hat{\chi}^{\mathrm{o}}(\mathbf{q})_{\ell, n}+\hat{\chi}^{\mathrm{o}}\left(\mathbf{q}^{*}\right)_{\ell, n-1}\right]
$$

using both identities in (5.21). We shall give density plots of several cases next, displaying the temperature dependence more clearly. However, it must

\footnotetext{
${ }^{9}$ In particular, one can start with the reflection symmetry about the direction of the $k_{j+3}$ grid-line and its action on the parallelograms $P\left(k_{j}, k_{j+1}\right)$ and $P\left(k_{j}^{\prime}, k_{j+1}^{\prime}\right)$. One arrives at $\left\langle\sigma \sigma^{\prime}\right\rangle_{\left[\ell, \ell^{\prime}, \ell^{\prime \prime}, \ell^{\prime \prime \prime}, \ell^{\prime \prime \prime \prime}\right]}=\left\langle\sigma \sigma^{\prime}\right\rangle_{\left[\ell^{\prime}, \ell, \ell^{\prime \prime \prime}, \ell^{\prime \prime \prime}, \ell^{\prime \prime}\right]}$. Replacing $j \rightarrow 1-j$ in (5.20) then completes the proof of the second identity in (5.21).
} 
be said that we can calculate $\hat{\chi}^{\mathrm{o}}(\mathbf{q})$ to very high precision in the cases shown, which fact is not clear from looking at these density plots.

We shall give plots both above the critical temperature $T_{\mathrm{c}},\left(k_{>} \equiv k<1\right)$, and below $T_{\mathrm{c}},\left(k_{<} \equiv 1 / k<1\right)$. The value of modulus $k$ corresponds to the row correlation length $(44)$

$$
\xi=1 /|\operatorname{arsinh}(1 / \sqrt{k})-\operatorname{arsinh}(\sqrt{k})|
$$

of the symmetric square-lattice Ising model for $T>T_{\mathrm{c}}$. For $T<T_{\mathrm{c}}$ the true value of this row correlation length is $\xi / 2$ with $\xi$ again given by (5.23). (44) 45)

At very low temperature, we only need to consider $\mathcal{S}_{\ell}$ for very small $\ell$. For $\ell, \ell^{\prime} \leqslant 2$ the joint probabilities $A_{m, m^{\prime}}\left(\ell, \ell^{\prime}\right)$ can be easily evaluated by hand as it only involves the calculation of areas of triangles and rectangles. For $k_{<}=.04847302$, which corresponds to $\xi \approx 1 / 2$, we find $\mathcal{S}_{\ell}<10^{-10}$ for $\ell>2$. The density plot for $1 / \hat{\chi}^{\mathrm{o}}(\mathbf{q})$ is shown in Fig. 9(a) for $-4 \pi \leqslant q_{x}, q_{y} \leqslant 4 \pi$ where $\mathbf{q}=\left(q_{x}, q_{y}\right)$. We find ten-fold symmetry, corresponding to the five-fold symmetry of the Penrose tiling.

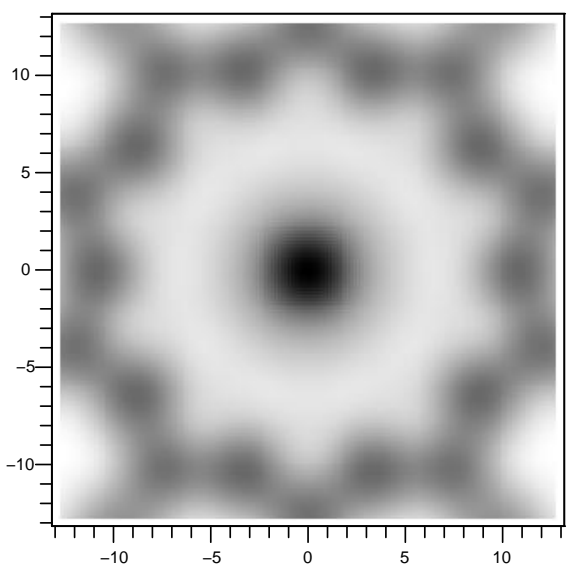

(a)

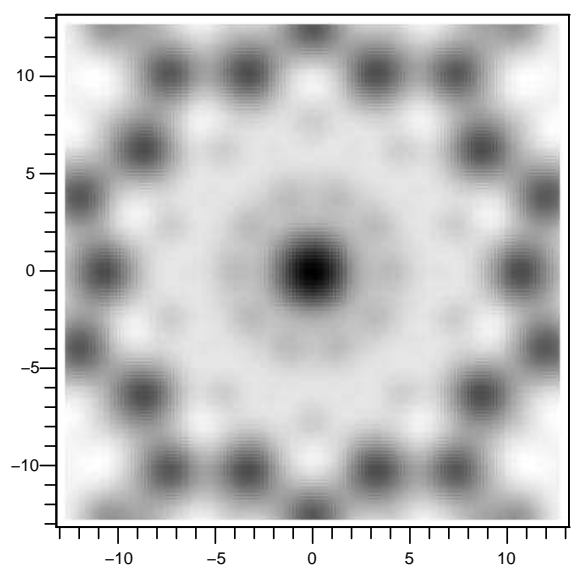

(b)

Fig. 9. Density plot of the q-dependent susceptibility showing $1 / \chi\left(q_{x}, q_{y}\right)$ for the $Z$ invariant Ising model on a Pentagrid Lattice (Penrose Tiles) at very low temperature: (a) $\xi \approx 0.5$, or $k_{<}=.04847302$; (b) $\xi \approx 1$, or $k_{<}=.2363562$, both for $T<T_{\mathrm{c}}$.

For $k_{<}=.2363562(\xi \approx 1)$, we find it necessary to consider all $\mathcal{S}_{\ell}$ for $\ell \leqslant 4$. As the temperature increases, larger and larger $\ell$ 's are needed. To 
evaluate the joint probability by hand is no longer feasible. To symbolically program the calculation for any values of $\ell$ and $\ell^{\prime}$ is highly nontrivial, as there are many different situations to take into account. It took us several months to sort out all cases, programming the calculation using Maple.

A density plot for $k_{<}=.2363562$ is shown in Fig. 9(b). Plots of $1 / \hat{\chi}^{\mathrm{o}}(\mathbf{q})$ for $\xi \approx 4$ and $\xi \approx 8$ are shown in Fig. 10(a) and Fig. 10(b), together with corresponding plots for the dual cases with $T>T_{\mathrm{c}}$ in Fig. 10(c) and Fig. 10(d). We can see clearly that the number of visible peaks increases as $T \rightarrow T_{\mathrm{c}}$ and that this effect is more pronounced as $T_{\mathrm{c}}$ is approached from above. In Fig. 11, a density plot for $1 / \hat{\chi}^{\mathrm{o}}(\mathbf{q})$ at $\xi=2$ is given for $-16 \pi \leqslant$ $q_{x}, q_{y} \leqslant 16 \pi$, and we can already see some evidence for the quasiperiodic pattern of the q-dependent susceptibility in the full $\left(q_{x}, q_{y}\right)$-plane.

\subsection{The q-dependent susceptibility $\chi^{\mathrm{e}}(\mathrm{q})$}

Finally, we show that the $\mathbf{q}$-dependent susceptibility $\hat{\chi}^{\mathrm{e}}(\mathbf{q})$ of the even sublattice is identical to $\hat{\chi}^{\mathrm{o}}(\mathbf{q})$ of the odd sublattice. From Tables III(a) and III(b), we find there are 16 different even configurations for $P\left(k_{j}, k_{j+1}\right)$. The corresponding 16 regions are plotted in the unit square with $\{\alpha\}$ and $\{\beta\}$ along the axes in Fig. 12(a). The regions for the 8 odd spin configurations of $P\left(k_{j}+1, k_{j+1}+1\right)$ are also plotted with $\{\alpha\}$ and $\{\beta\}$ as axes in Fig. 12(b). It is easy to see that after inverting one of the squares, the two figures are identical up to labeling.

Furthermore, looking at Fig. 12(b), we see that the five disjoint regions $2, \cdots, 6$ become connected, if we impose periodic boundary conditions on the square. This "wrapping on a torus" is consistent with moving the slices as discussed below Eq. (5.11). We next compare how the even regions in Fig. 12 (a) relate under the same periodic boundary conditions, to see if this is somehow true here also. As first examples we look at the two even configurations $\mathrm{e}(7)$ and $\mathrm{e}(13)$ and find that they have the same number of sites, and their difference vectors are related by

$$
\delta \vec{K}^{\mathrm{e}, 7}=(0,-1,1)+\delta \vec{K}^{\mathrm{e}, 13}
$$

For the other regions, we similarly find equal numbers of sites and

$$
\begin{aligned}
& \delta \vec{K}^{\mathrm{e}, 10}=(1,-1,0)+\delta \vec{K}^{\mathrm{e}, 1}, \quad \delta \vec{K}^{\mathrm{e}, 3+m}=(1,-1,0)+\delta \vec{K}^{\mathrm{e}, 14+m}, \\
& \delta \vec{K}^{\mathrm{e}, 6}=(0,-1,1)+\delta \vec{K}^{\mathrm{e}, 1}, \quad \delta \vec{K}^{\mathrm{e}, 8+n}=(0,-1,1)+\delta \vec{K}^{\mathrm{e}, 15+n},
\end{aligned}
$$




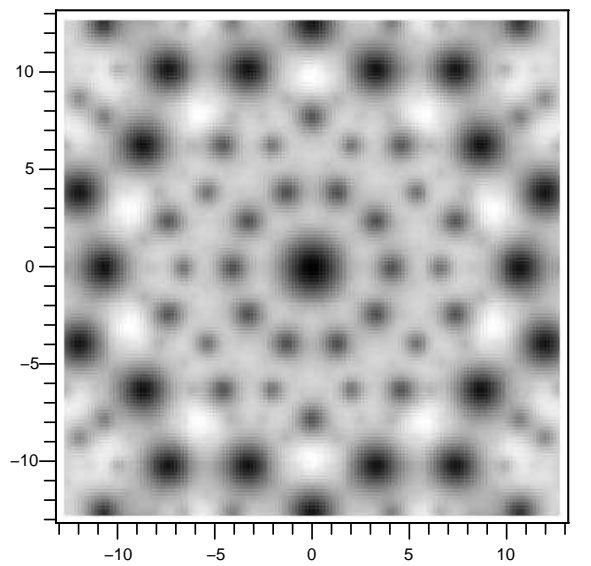

(a)

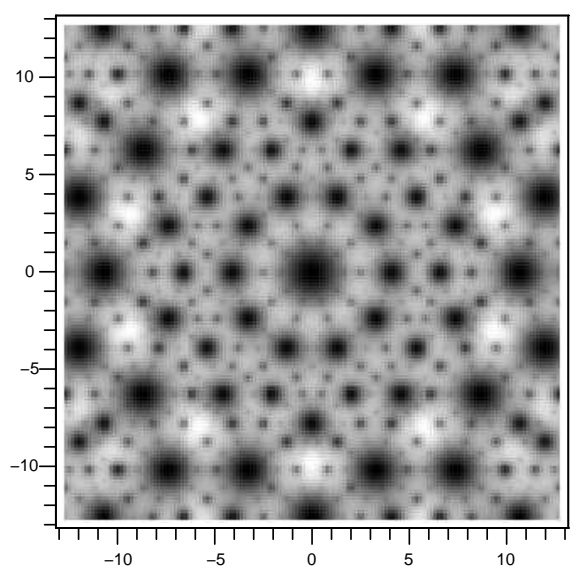

(c)

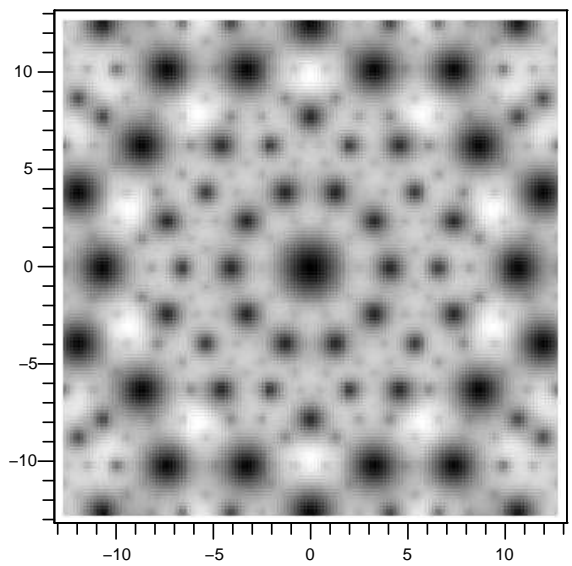

(b)

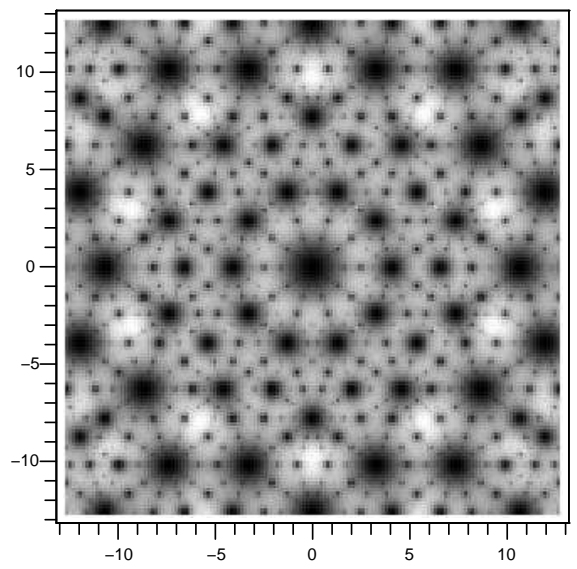

(d)

Fig. 10. Density plots of the q-dependent susceptibility, plotting $1 / \chi(\mathbf{q})$ versus $q_{x}$ and $q_{y}$ : (a) $k_{<}=.7018662$ or $\xi \approx 4$; (b) $k_{<}=.8379187$ or $\xi \approx 8$, both for $T<T_{\mathrm{c}}$. Two corresponding plots for the dual models, with $T>T_{\mathrm{c}}$ and identical values of $k_{>}$and $\xi$, are given in (c) and (d). 


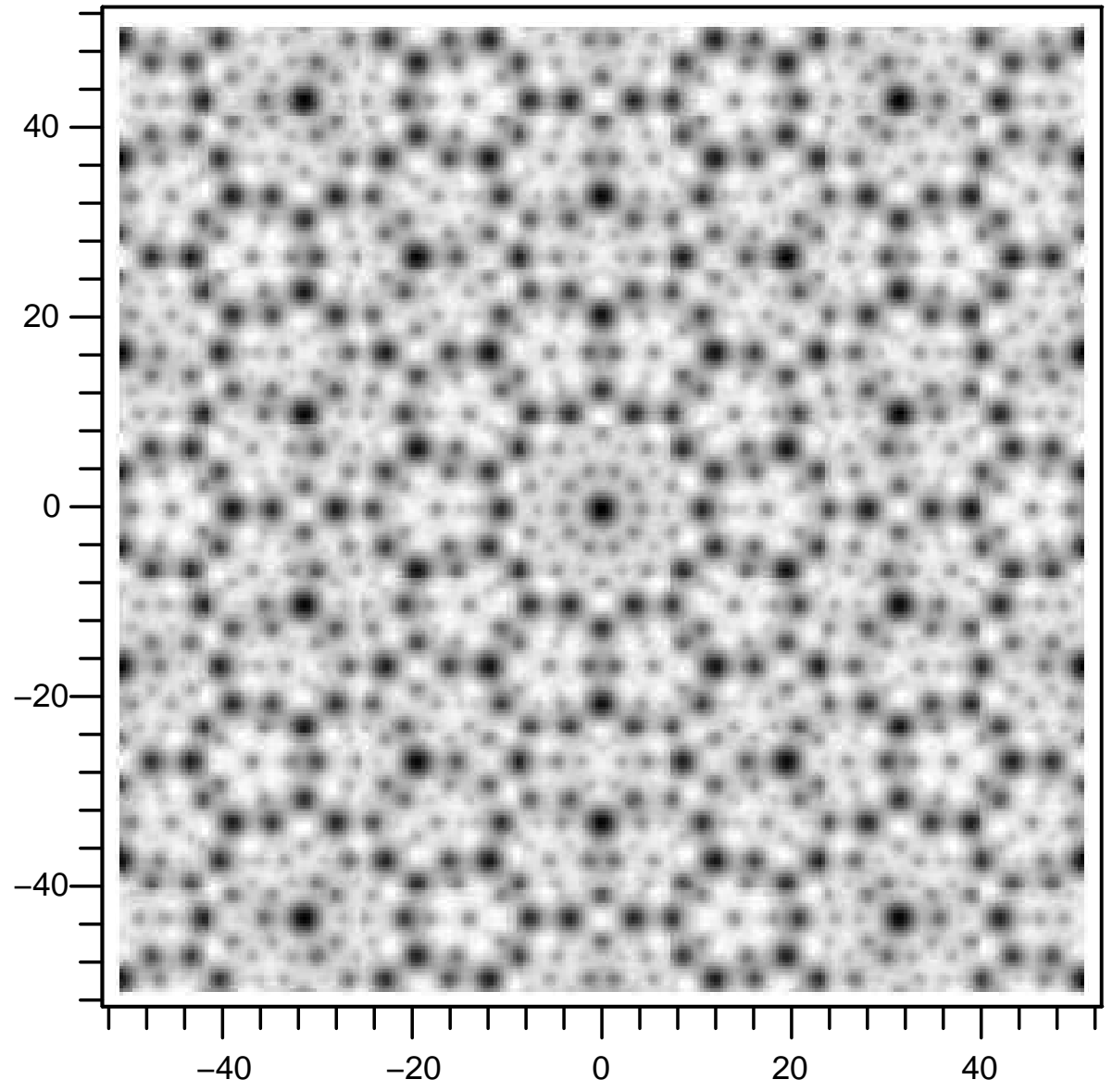

Fig. 11. Density plot of the $\mathbf{q}$-dependent susceptibility showing $1 / \chi(\mathbf{q})$ at $k_{<}=.4912758$ or $\xi \approx 2$ and $T<T_{\mathrm{c}}$. 
where $m=0,1,2$ and $n=0,1$.

The difference vectors of the odd configurations in Fig. 12(b) are also related to those of the even configurations in Fig. 12(a), i.e.

$$
\begin{aligned}
& \delta \vec{K}^{\mathrm{e}, 1}=(1,-1,1)-\delta \vec{K}^{\mathrm{o}, 2}, \quad \delta \vec{K}^{\mathrm{e}, 2}=(1,-1,1)-\delta \vec{K}^{\mathrm{o}, 1} \\
& \delta \vec{K}^{\mathrm{e}, 11+n}=(0,1,0)-\delta \vec{K}^{\mathrm{o}, 8-n}, \quad n=0, \cdots, 5 .
\end{aligned}
$$

If the dependences of difference vectors on the values of $\{\alpha\}$ and $\{\beta\}$ are included in the equations, we may relate the even spins in $P\left(k_{j}, k_{j+1}\right)$ with the odd spins in $P\left(k_{j}+1, k_{j+1}+1\right)$ by

$$
\delta \vec{K}^{\mathrm{e}}[\{\alpha\},\{\beta\}]=(1,-1,1)-\left(\delta_{2},-\delta_{2}-\delta_{4}, \delta_{4}\right)-\delta \vec{K}^{\mathrm{o}}[\{-\alpha\},\{-\beta\}],
$$

where $1-\{x\}=\{-x\}$, for $x$ not an integer, and

$$
\delta_{2}=\left\lfloor 1-\{\alpha\}+p^{-1}\right\rfloor, \quad \delta_{4}=\left\lfloor 1-\{\beta\}+p^{-1}\right\rfloor .
$$

Consider the pentagrid with $\gamma_{j} \rightarrow-\gamma_{j}$ and denote its parallelograms by $\bar{P}\left(k_{j}, k_{j+1}\right)$ such that

$$
\bar{\alpha}\left(-k_{j+1}\right)=-\alpha\left(k_{j+1}\right), \quad \bar{\beta}\left(-k_{j}\right)=-\beta\left(k_{j}\right) .
$$

Then it is easy to show that

$$
\delta_{2}+\left\lfloor-\alpha\left(k_{j+1}\right)\right\rfloor=\left\lfloor\bar{\alpha}\left(1-k_{j+1}\right)\right\rfloor, \quad \delta_{4}+\left\lfloor-\beta\left(k_{j}\right)\right\rfloor=\left\lfloor\bar{\beta}\left(1-k_{j}\right)\right\rfloor .
$$

Now, the integer vectors of even spins in $P\left(k_{j}, k_{j+1}\right)$ given by (4.9), (4.3) and (4.5) can be shown to relate to the integer vectors of the odd spins in $\bar{P}\left(1-k_{j}, 1-k_{j+1}\right)$ by

$$
\vec{K}^{\mathrm{e}}\left(\boldsymbol{\epsilon}^{\prime}\right)=(1,1,1,1,1)-\vec{K}^{\mathrm{o}}(\boldsymbol{\epsilon}) .
$$

This equation is consistent with the fact that the index of the odd spins is either 1 or 3 , while the index of the even spins is either 2 or 4 . Since the even spins in the original pentagrid are related to the odd spins in a different pentagrid, and the joint probabilities are independent of shifts, we have shown that the susceptibility of the even sublattice is identically the same as the one of the odd sublattice. 


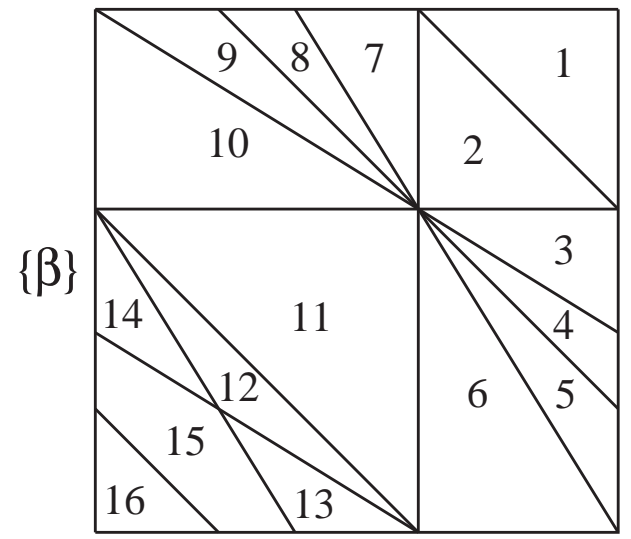

$\{\alpha\}$

(a)

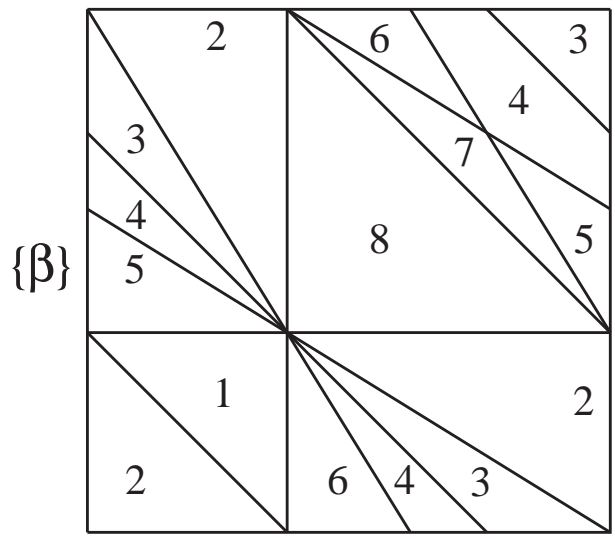

$\{\alpha\}$

(b)

Fig. 12. (a) The sixteen even spin configurations of $P\left(k_{j}, k_{j+1}\right)$ plotted with $\{\alpha\}$ and $\{\beta\}$ as the horizontal and vertical axis. (b) The eight odd spin configurations of $P\left(k_{j}+1, k_{j+1}+1\right)$ plotted with $\{\alpha\}$ and $\{\beta\}$.

\section{Conclusions and Final Remarks}

In this paper we have presented a systematic way of evaluating the averaged pair-correlation function of a $Z$-invariant ferromagnetic Ising model with spins on half the sites of a Penrose tiling and Ising interactions across the diagonals of the rhombuses.

Next, we have found that the q-dependent susceptibility of this model is a superposition of incommensurate everywhere-dense peaks, though not many peaks are visible at temperatures very far away from $T_{\mathrm{c}}$. For $T<T_{\mathrm{c}}$ these peaks add a diffuse background to the Bragg peaks due to the spontaneous magnetization. Since the $S_{\ell}$ in (5.22) consists of $4 \ell$ terms of the same order of magnitude, we compare their contributions. We find that the number of peaks of $S_{\ell}$ increases as $\ell$ increases, but that the numbers at fixed $\ell$ are almost independent of temperature, even though the magnitudes of the peaks change as the temperature varies.

As $T \rightarrow T_{\mathrm{c}}$, the correlations decay more and more slowly, so the $S_{\ell}$ 's increase, and more and more of these $S_{\ell}$ 's are to be included in the numerical evaluation of the q-dependent susceptibility, which accounts for the everincreasing number of peaks. This is unlike the behavior of the Fibonacci Ising models, considered earlier, ${ }^{(53[54)}$ where the ferromagnetic aperiodic Fibonacci 
lattice behaves almost like the regular Ising model.

Moreover, the q-dependent susceptibility is not a periodic function of $q_{x}$ or $q_{y}$. This behavior is different from that of aperiodic models defined on regular lattices. $\stackrel{(53)}{(5)}$ This is because, when the lattice is aperiodic, we cannot separate the average of the correlation functions from the exponential (or cosine) terms, which contain the information about the lattice structure, as can be seen from (5.19) and (5.20).

At $T_{\mathrm{c}}$, the q-dependent susceptibility has everywhere-dense divergences with the Ising exponent $7 / 4$, but is still locally integrable. Away from $T_{\mathrm{c}}$ the $\chi(\mathbf{q})$ is a continuous function. The Bragg peaks below $T_{\mathrm{c}}$ form a set of everywhere-dense Dirac delta functions of various strengths, but their sum is also locally integrable. These are strange objects and de Bruijn initiated their mathematical study. ${ }^{(65)}$

One of the main results of the current paper is that it provides a new method for doing calculations of probabilities on Penrose tilings. In Section 4, the calculation of the joint probability of the configurations of two parallelograms on the pentagrid is reduced to linear programming.

Penrose tilings may be obtained by projecting certain subsets of the $\mathbb{Z}^{5}$ lattice into the plane. ${ }^{(5)}$ The frequencies of the different types of vertices are then given as areas in the orthogonal spaces. ${ }^{(5)}$ This method, which is known as the cut-and-project method, has been applied and generalized by

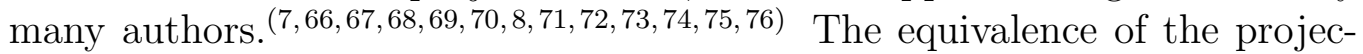
tion method and a generalized grid method has been demonstrated. (66],67][68) The positions of the Bragg peaks have been worked out, $, 769,70|8| 71 \mid 72)$ together with the values of probabilities of local configurations. The Penrose tilings can even be obtained from projections in a four-dimensional root lattice. $\frac{(75,76)}{17}$

It would be interesting to obtain results for joint probabilities similar to ours also by cut-and-project methods. This would generalize the windowing method of Baake and Grimm. ${ }^{(77)}$ We have not pursued this here, as our model is defined in terms of rapidity lines on the pentagrid.

Another possible generalization of our work is to consider Penrose tilings that are periodic in either one or both directions. Finite approximants to the aperiodic Penrose tiling have been constructed through periodic pentagrids or projection methods. $\stackrel{(78,79,80,73)}{7,}$

The mathematician Robinson has brought to our attention an exercise in the book by Grünbaum and Shephard ${ }^{(81)}$ where a tiling with Penrose rhombuses can be cut into patches, and then converted into an aperiodic 
set of 24 Wang's tiles. We have found that each patch in the Penrose tiling described in the exercise is in fact the image of a parallelogram under the mapping (2.5). Thus these 24 configurations of the parallelogram can be easily converted into Wang's tiles.

Finally, in our previous studies ${ }^{(53)}$ we have examined the $q$-dependent susceptibility $\chi(\mathbf{q})$ of some quasiperiodic Ising models on the square lattice defined in terms of Fibonacci sequences. It may be of interest to study models based on other sequences such as the aperiodic sequences studied by de Bruijn ${ }^{(61)}$ or Tracy. ${ }^{(50)}$ We may ask what effect this has on the mixed interaction cases and also if it makes any difference for purely ferromagnetic models.

\section{ACKNOWLEDGMENTS}

An earlier version of this work has been presented to Professor M. E. Fisher at a conference in honor of his seventieth birthday. We are most thankful to Dr. M. Widom for his interest in using exactly solvable models to study quasicrystals, which led us to start this work. We also thank Dr. M. Baake, Dr. U. Grimm, and Dr. E. A. Robinson for providing us with many useful references. This work has been supported by NSF Grant PHY 01-00041.

\section{REFERENCES}

1. D. Shechtman, I. Blech, D. R. Gratias, and J. W. Cahn, Metallic phase with longrange orientational order and no translational symmetry, Phys. Rev. Lett. 53:19511953 (1984).

2. R. Penrose, Tilings and quasi-crystals; a non-local growth problem?, in Introduction to The Mathematics of Quasicrystals, Aperiodicity and Order, Vol. 2, M. V. Jarić, ed. (Academic Press, Boston, 1989), pp. 53-79.

3. R. Penrose, The rôle of aesthetics in pure and applied mathematical research, Bull. Inst. Math. Appl. 10:266-271 (1974).

4. R. Penrose, Pentaplexity: A class of non-periodic tilings of the plane, The Mathematical Intelligencer 2:32-37 (1979), [reprinted from Eureka No. 39].

5. N. G. de Bruijn, Algebraic theory of Penrose's non-periodic tilings of the plane. I, Indagationes Mathematicae 84:38-52 (1981); —. II, ibid. 84:53-66 (1981).

6. A. L. Mackay, De nive quinquangula: On the pentagonal snowflake, Kristallografiya 26:910-919 (1981) [Sov. Phys. Crystallogr. 26:517-522 (1981)].

7. A. L. Mackay, Crystallography on the Penrose pattern, Physica A 114:609-613 (1982). 
8. D. Levine and P. J. Steinhardt, Quasicrystals: A new class of ordered structures, Phys. Rev. Lett. 53:2477-2480 (1984).

9. M. Baake, U. Grimm, and R. V. Moody, Die verborgene Ordnung der Quasikristalle, Spektrum der Wissenschaft, Heft 02 (Februar 2002) 64-74, [in German, English translation: What is Aperiodic Order?, Preprint math.HO/0203252.

10. C. Janot, Quasicrystals: A Primer, 2nd Ed. (Clarendon Press, Oxford, 1994).

11. C. L. Henley, Quasicrystal order, its origins and its consequences: A survey of current models, Comments Cond. Mat. Phys. 13:59-117 (1987).

12. M. Baake, A guide to mathematical quasicrystals, in Quasicrystals - An Introduction to the Structure, Physical Properties, and Applications, Material Science, Vol. 55, J.B. Suck, M. Schreiber, and P. Häußler, eds., (Springer, Berlin, 2002), pp. 17-48.

13. V. E. Korepin, Eight-vertex model of the quasicrystal, Phys. Lett. A 118:285-287 (1986).

14. R. J. Baxter, Solvable eight vertex model on an arbitrary planar lattice, Phil. Trans. R. Soc. Lond. A 289:315-346 (1978).

15. V. E. Korepin, Completely integrable models in quasicrystals, Commun. Math. Phys. 110:157-171 (1987).

16. N. V. Antonov and V. E. Korepin, Critical properties and correlation functions of the eight-vertex model on a quasicrystal, Zap. Nauch. Semin. LOMI 161:13-23 (1987) [J. Sov. Math. 46:2058-2065 (1989)].

17. N. V. Antonov and V. E. Korepin, Critical properties of completely integrable spin models in quasicrystals, Teor. Mat. Fiz. 77:402-411 (1988) [Theor. Math. Phys. 77:1282-1288 (1988)].

18. T. C. Choy, Ising models on two-dimensional quasi-crystals: Some exact results, Intern. J. Mod. Phys. B 2:49-63 (1988).

19. M. Baake, U. Grimm, and R. J. Baxter, A critical Ising model on the labyrinth, Intern. J. Mod. Phys. B 8:3579-3600 (1994).

20. U. Grimm, M. Baake, and H. Simon, Ising spins on the labyrinth, in Proc. of the 5th International Conference on Quasicrystals, C. Janot and R. Mosseri, eds. (World Scientific, Singapore, 1995), pp. 80-83.

21. U. Grimm and M. Baake, Aperiodic Ising models, in The Mathematics of Long-Range Aperiodic Order, R. V. Moody, ed. (Kluwer, Dordrecht, 1997), pp. 199-237.

22. H. Aoyama and T. Odagaki, Eight-parameter renormalization group for Penrose lattices, J. Stat. Phys. 48:503-511 (1987).

23. G. Amarendra, G. Ananthakrishna, and G. Athithan, Critical behavior of the Ising model on a two-dimensional Penrose lattice, Europhys. Lett. 5:181-184 (1988).

24. S. M. Bhattacharjee, J.-S. Ho, and J. A. Y. Johnson, Translational invariance in critical phenomena: Ising model on a quasi-lattice, J. Phys. A 20:4439-4448 (1987).

25. Y. Okabe and K. Niizeki, Monte Carlo simulation of the Ising model on the Penrose lattice, J. Phys. Soc. Jpn. 57:16-19 (1988).

26. Y. Okabe and K. Niizeki, Duality in the Ising model on the quasicrystals, J. Phys. Soc. Jpn. 57:1536-1539 (1988).

27. E. S. Sørensen, M. V. Jarić, and M. Ronchetti, Ising model on the Penrose lattice: Boundary conditions, Phys. Rev. B 44:9271-9282 (1991).

28. L.-H. Tang and M. V. Jarić, Equilibrium quasicrystal phase of a Penrose tiling model, 
Phys. Rev. B 41:4524-4546 (1990), see p. 4537.

29. O. Redner and M. Baake, Invaded cluster algorithm for critical properties of periodic and aperiodic planar Ising models, J. Phys. A 33:3097-3109 (2000).

30. R. Abe and T. Dotera, High temperature expansion for the Ising model on the Penrose lattice, J. Phys. Soc. Jpn. 58:3219-3226 (1989).

31. T. Dotera and R. Abe, High temperature expansion for the Ising model on the dual Penrose lattice, J. Phys. Soc. Jpn. 59:2064-2077 (1990).

32. P. Repetowicz, Finite-lattice expansion for the Ising model on the Penrose tiling, $J$. Phys. A 35:7753-7772 (2002).

33. H. Simon, M. Baake, and U. Grimm, Lee-Yang zeros for substitutional systems, in Proc. of the 5th International Conference on Quasicrystals, C. Janot and R. Mosseri, eds., (World Scientific, Singapore, 1995), pp. 100-103.

34. H. Simon and M. Baake, Lee-Yang zeros in the scaling region of a two-dimensional quasiperiodic Ising model, J. Phys. A 30:5319-5327 (1997).

35. P. Repetowicz, U. Grimm, and M. Schreiber, Planar quasiperiodic Ising models, Mat. Science Eng. A 294-296:638-641 (2000).

36. H. Au-Yang and J. H. H. Perk, Susceptibility calculations in periodic and quasiperiodic planar Ising models, Physica A 321:81-89 (2003).

37. D. Levine and P. J. Steinhardt, Quasicrystals. I. Definition and structure, Phys. Rev. B 34:596-616 (1986).

38. J. E. S. Socolar and P. J. Steinhardt, Quasicrystals. II. Unit-cell configurations, Phys. Rev. B 34:617-647 (1986).

39. T. C. Lubensky, J. E. S. Socolar, P. J. Steinhardt, P. A. Bancel, and P. A. Heiney, Distortion and peak broadening in quasicrystal diffraction patterns, Phys. Rev. Lett. 57:1440-1443 (1986).

40. A. Hof, Diffraction of aperiodic structures at high temperatures, J. Phys. A 28:57-62 (1995).

41. B. Kaufman and L. Onsager, Crystal statistics. III. Short-range order in a binary Ising lattice, Phys. Rev. 76:1244-1252 (1949).

42. M. E. Fisher, The susceptibility of the plane Ising model, Physica 25:521-524 (1959).

43. E. W. Montroll, R. B. Potts, and J. C. Ward, Correlations and spontaneous magnetization of the two-dimensional Ising model, J. Math. Phys. 4:308-322 (1963).

44. B. M. McCoy and T. T. Wu, The Two-Dimensional Ising Model (Harvard Univ. Press, Cambridge, Mass., 1973).

45. T. T. Wu, B. M. McCoy, C. A. Tracy, and E. Barouch, Spin-spin correlation functions for the two-dimensional Ising model: Exact theory in the scaling region, Phys. Rev. B 13:316-374 (1976).

46. D. B. Abraham, Pair function for the rectangular Ising ferromagnet, Commun. Math. Phys. 60:181-191 (1978).

47. J. H. H. Perk, Quadratic identities for Ising correlations, Phys. Lett. A 79:3-5 (1980).

48. H. Au-Yang and J. H. H. Perk, Critical correlations in a $Z$-invariant inhomogeneous Ising model, Physica A 144:44-104 (1987).

49. C. A. Tracy, Universality class of a Fibonacci Ising model, J. Stat. Phys. 51:481-490 (1988).

50. C. A. Tracy, Universality classes of some aperiodic Ising models, J. Phys. A 21:L603- 
L605 (1988).

51. H. Au-Yang and B. M. McCoy, Theory of layered Ising models. II. Spin correlation functions parallel to the layering, Phys. Rev. B 10:3885-3905 (1974).

52. B. M. McCoy and T. T. Wu, Theory of Toeplitz determinants and spin correlations of the two-dimensional Ising model. II, Phys. Rev. 155:438-452 (1967).

53. H. Au-Yang, B.-Q. Jin, and J. H. H. Perk, Wavevector-dependent susceptibility in quasiperiodic Ising models, J. Stat. Phys. 102:501-543 (2001).

54. H. Au-Yang and J. H. H. Perk, Wavevector-dependent susceptibility in aperiodic planar Ising models, in MathPhys Odyssey 2001: Integrable Models and Beyond, M. Kashiwara and T. Miwa, eds. (Birkhäuser, Boston, 2002), pp. 1-21.

55. X.-P. Kong, Wave-Vector Dependent Susceptibility of the Two-Dimensional Ising Model, (Ph. D. Thesis, State University of New York at Stony Brook, September 1987).

56. P. W. Stephens, The icosahedral glass model, in Extended Icosahedral Structures, Aperiodicity and Order, Vol. 3, M. V. Jarić and D. Gratias, eds. (Academic Press, Boston, 1989), pp. 37-104.

57. M. Gardner, Extraordinary nonperiodic tiling that enriches the theory of tiles, Scientific American 236\#1:110-121 (January 1977).

58. F. Y. Wu, Ising model with four-spin interactions, Phys. Rev. B 4:2312-2314 (1971).

59. L. P. Kadanoff and F. J. Wegner, Some critical properties of the eight-vertex model, Phys. Rev. B 4:3989-3993 (1971).

60. H. Au-Yang and J. H. H. Perk, Correlation functions and susceptibility in the $Z$ invariant Ising model, in MathPhys Odyssey 2001: Integrable Models and Beyond, M. Kashiwara and T. Miwa, eds. (Birkhäuser, Boston, 2002), pp. 23-48.

61. N. G. de Bruijn, Sequences of zeros and ones generated by special production rules, Indagationes Mathematicae 84:27-37 (1981).

62. G. H. Hardy and E. M. Wright, An Introduction to the Theory of Numbers, 4th edition, (Oxford University Press, London, 1960), Ch. XXIII Kronecker's Theorem.

63. H. Au-Yang and J. H. H. Perk, New results for susceptibilities in planar Ising models, Int. J. Mod. Phys. B 16:2089-2095 (2002).

64. H. Au-Yang and J. H. H. Perk, Susceptibility calculations in periodic and quasiperiodic planar Ising models, Physica A 321:81-89 (2003).

65. N. G. de Bruijn, Quasicrystals and their Fourier transform, Indagationes Mathematicae 89:123-152 (1986).

66. P. Cramer and R. Neri, On periodic and non-periodic space fillings of $\mathbb{E}^{m}$ obtained by projection, Acta Crystallogr. A 40:580-587 (1984).

67. F. Gähler and J. Rhyner, Equivalence of the generalised grid and projection methods for the construction of quasiperiodic tilings, J. Phys. A 19:267-277 (1986).

68. V.E. Korepin, F. Gähler, and J. Rhyner, Quasiperiodic tilings: A generalized gridprojection method, Acta Crystallogr. A 44:667-672 (1988).

69. M. Duneau and A. Katz, Quasiperiodic patterns, Phys. Rev. Lett. 54:2688-2691 (1985).

70. P. A. Kalugin, A. Yu. Kitaev, and L. S. Levitov, $\mathrm{Al}_{0.86} \mathrm{Mn}_{0.14}$; A six-dimensional crystal, P'isma Zh. Eksp. Teor. Fiz. 41:119-121 (1985) [JETP Lett. 41:145-149 (1985)].

71. M. V. Jarić, Diffraction from quasicrystals: Geometric structure factor, Phys. Rev. 
B 34:4685-4698 (1986).

72. V. Elser, The diffraction pattern of projected structures, Acta Crystallogr. A 42:3643 (1986).

73. P. Repetowicz, U. Grimm, and M. Schreiber, High-temperature expansion for Ising models on quasiperiodic tilings, J. Phys. A 32:4397-4418 (1999).

74. A. N. Rogers, C. Richard, and A. J. Guttmann, Self-avoiding walks and polygons on quasiperiodic tilings, J. Phys. A 36:6661-6673 (2003).

75. M. Baake, P. Kramer, M. Schlottmann, and D. Zeidler, Planar patterns with fivefold symmetry as sections of periodic structures in 4-space, Intern. J. Mod. Phys. A 15-16:2217-2268 (1990).

76. M. Baake, D. Joseph, P. Kramer, and M. Schlottmann, Root lattices and quasicrystals, J. Phys. A 23:L1037-L1041 (1990).

77. M. Baake and U. Grimm, Combinatorial problems of (quasi-)crystallography, in Quasicrystals - Structure and Physical Properties, H.-R. Trebin, ed. (Wiley-VCH, New York, 2003), pp. 160-171.

78. H. Tsunetsugu, T. Fujiwara, K. Ueda, and T. Tokihiro, Eigenstates in 2-dimensional Penrose tiling, J. Phys. Soc. Jpn. 55:1420-1423 (1986).

79. H. Tsunetsugu, T. Fujiwara, K. Ueda, and T. Tokihiro, Electronic properties of the Penrose lattice. I. Energy spectrum and wave functions, Phys. Rev. B 43:8879-8891 (1991).

80. H. Tsunetsugu and K. Ueda, Electronic properties of the Penrose lattice. II. Conductance at zero temperature, Phys. Rev. B 43:8892-8902 (1991).

81. B. Grünbaum and G.C. Shephard, Tilings and Patterns (W.H. Freeman and Co., New York, 1987), Ch. 11, Wang Tiles. 